J. R. Dennison and A. L. Ritter, "Application of (e,2e) Spectroscopy to the Electronic Structure of Valence Electrons in Crystalline and Amorphous Solids, J. Electron Spec. Rel. Phenom., 77, 99-142 (1996). DOI: 10.1016/0368-2048(95)02496-4

\title{
Review
}

\section{Application of (e,2e) spectroscopy to the electronic structure of valence electrons in crystalline and amorphous solids}

\author{
J.R. Dennison ${ }^{a}$ and A.L. Ritter ${ }^{b}$ \\ a Department of Physics, Utah State University, Logan, UT 84322-4415 \\ b Department of Physics, Virginia Tech, Blacksburg, VA 24061
}

(Received

\begin{abstract}
This review presents theoretical and experimental aspects of $(e, 2 e)$ spectroscopy specific to the study of crystalline and amorphous solids. The cross section for $(\mathrm{e}, 2 \mathrm{e})$ scattering is proportional to the spectral momentum density of the ejected electron under certain approximations. The theoretical framework for interpreting (e,2e) measurements is summarized here and general properties of the spectral momentum density of solids are discussed. Different designs of the (e,2e) spectrometer are described and sample preparation techniques are briefly reviewed. A summary of recent $(e, 2 e)$ experiments on the electronic structure of valence electrons in graphite, ion sputtered and evaporated amorphous carbon, and aluminum-aluminum oxide films is given. The review concludes with a discussion of an "ideal" (e,2e) spectrometer for studying bulk properties of solids.
\end{abstract}




\section{CONTENTS}

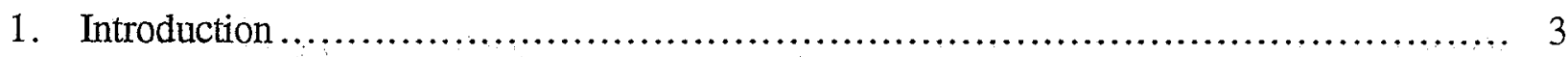

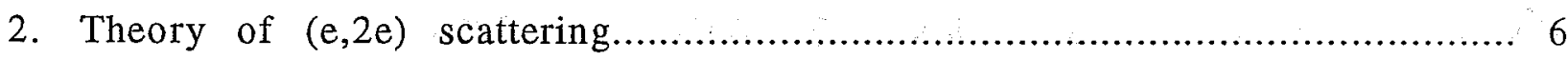

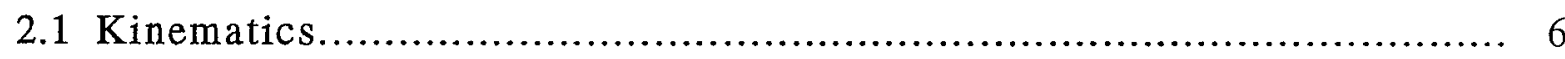

2.2 Cross section ....................................................................... 7

2.3 Justification of approximations.......................................................... 8

2.4 Spectral momentum density of crystalline solids.......................................... 10

2.5 Spectral momentum density of amorphous solids........................................ 11

2.6 Integrals of the spectral momentum density................................................ 13

2.7 Implications of multiple scattering for solids..................................... 14

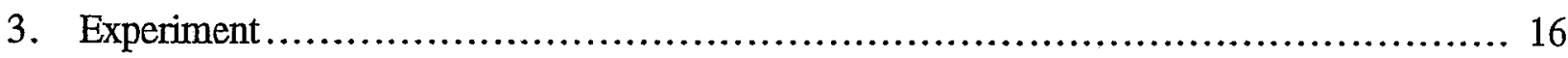

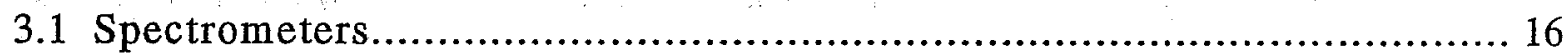

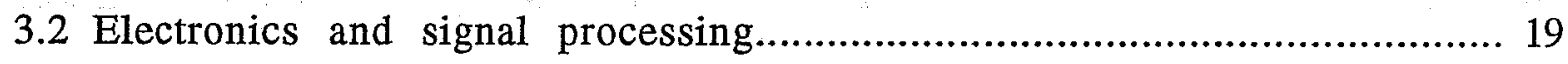

3.3 Sample preparation ................................................................. 20

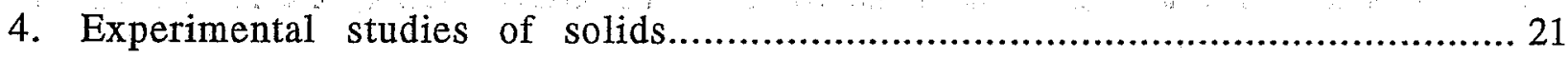

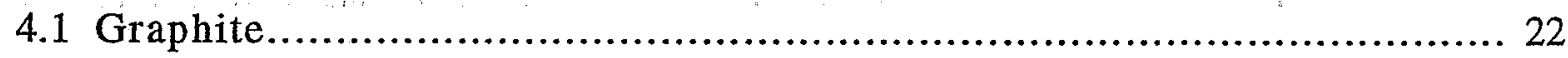

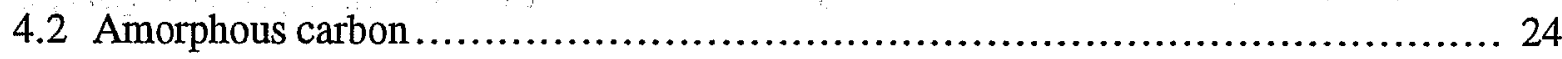

4.2.1. Evaporated amorphous carbon

4.2.2. Ion sputtered amorphous carbon

4.2.3. Models of amorphous carbon

4.3 Aluminum/aluminum oxide.......................................................... 31

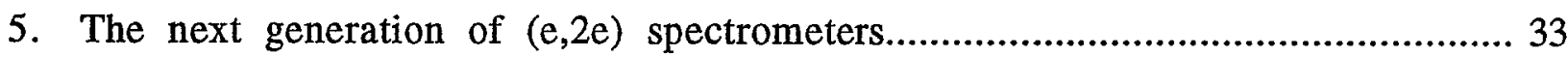

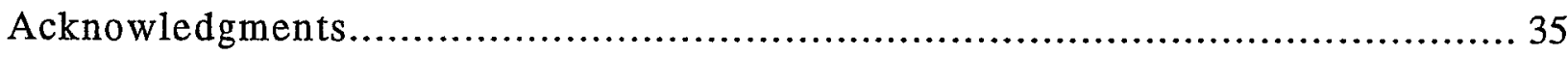

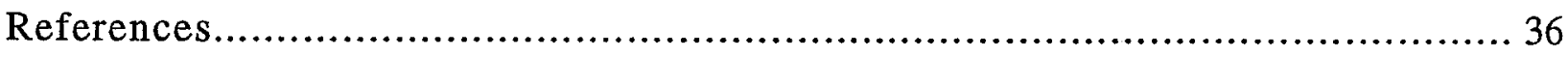

Appendix: Functional dependence of $(\mathrm{e}, 2 \mathrm{e})$ count rate.................................................... 41 


\section{INTRODUCTION}

The potential power of (e,2e) spectroscopy to elucidate the electronic structure of solids has been recognized for almost thirty years [1,2], but successful application of this technique to solids has become a reality only in the last decade [3]. The central importance of this technique is that the $(e, 2 e)$ scattering cross section is proportional to the spectral momentum density (SMD) of the target; that is, the (e,2e) coincidence count rate is a direct measure of the probability of finding an electron in the solid with given energy and momentum. To illustrate this, the SMD of a hypothetical solid with a nearly-free electron valence band and an atomic-like core band is shown in Fig. 1. The valence band disperses quadratically with momentum up to a small energy gap at the Brillouin zone boundary and continues the quadratic dispersion in the second Brillouin zone. The SMD is nearly constant except near a zone boundary where it falls off rapidly (for a given band) in the neighboring zone. In a metal the SMD is cut off at the Fermi energy. The atomic-like core band is nearly dispersionless and the SMD mirrors the momentum density of the atomic level. Significantly, the $(e, 2 e)$ technique can be applied to amorphous as well as crystalline systems.

The difficulty in applying the technique is that the cross section and, therefore, the count rate is very small. Other techniques, such as photoemission and positron annihilation, can measure integrals of the SMD more expeditiously and with higher precision. In crystalline solids, where there is considerable symmetry in the electronic structure, one can often infer important features of the SMD from the integrals. But in disordered solids, where the translational symmetry is absent, the integrated SMD is typically smooth and featureless and, here, (e,2e) spectroscopy can make significant contributions to our understanding of these materials.

The application of $(e, 2 e)$ spectroscopy to solids has progressed in three distinguishable stages. The first experiments by Amaldi et al. on polyvinyl-formal film [4] and Camilloni et al. on carbon films [5] demonstrated that the technique could be applied to solids. The resolution $(\sim 100 \mathrm{eV})$ was sufficient to distinguish core and valence shells, but was not sufficient to resolve features of the valence shell. A series of experiments on carbon [6,7], collodion [6], formvar [8], and aluminum $[7,9,10]$ also could not resolve details of the valence shell, but the energy resolution improved by an 
order of magnitude during this period. The first stage in the application of $(e, 2 e)$ spectroscopy to the valence band structure of solids (reviewed in Refs. 11-13) ends with the energy resolution on the order of a typical solid valence band width.

The second stage began with an experiment by Ritter et al. on evaporated amorphous carbon films which resolved features of the valence band for the first time [3]. Three prime factors contributed to the success of this experiment [14]. First, the improved energy resolution (6 to $8 \mathrm{eV})$ and the fortuitously broad band width of amorphous carbon $(20 \mathrm{eV})$ made it possible to resolve valence bands derived from molecular $\sigma$ and $\pi$ orbitals. Second, the use of ultra high vacuum technology contributed to the stability of the target under relatively intense electron beam bombardment over the necessarily long period of data accumulation. Finally, the incident beam energy was approximately a factor of two higher than earlier experiments. This reduced multiple scattering which was a far more deleterious factor in (e,2e) experiments on solids than had been anticipated. The group at Virginia Tech followed this first experiment with studies of crystalline graphite [15] and diamondlike amorphous carbon [16]. The results on evaporated amorphous carbon were confirmed with higher resolution by Hayes et al. [17] and Lower et al. [18]. This second stage was marked by the first, clear evidence that $(e, 2 e)$ spectroscopy could provide new, illuminating information regarding the electronic structure of valence electrons in crystalline and amorphous solids.

The major experimental difficulty encountered by groups in the first and second stages was the length of time required to take a complete set of data on one sample. This low data rate was due to the small inherent cross section for $(\mathrm{e}, 2 \mathrm{e})$ scattering and the experimental practice of taking each energy and momentum point in serial order using single channel detectors. Stage three is marked by the advent of multichannel techniques permitting parallel collection of data over extended energy and momentum ranges, which significantly improves the data rate and facilitates significant increases in energy resolution [13,18-28; reviewed in 28]. In this review, emphasis is placed on stage two and stage three developments in the application of $(e, 2 \mathrm{e})$ spectroscopy to solids. More details 
about the earlier work can be found in previous reviews [11-13]. We will not discuss the application of (e,2e) spectroscopy to core levels [29-31] and surfaces [32].

As the technical hurdles to the application of $(e, 2 \mathrm{e})$ spectroscopy to solids are overcome, one would, of course, like to apply the technique to problems of interest in solid state physics. But, as with any new technique, it is important to apply it first to systems which are well understood before attempting to study those materials and questions which are conceptually more challenging. Systematic experimental errors can be detected more easily when the "correct" results are known. In addition to experimental sources of error, several assumptions and approximations in scattering theory are hidden in the simple proportionality between the $(e, 2 e)$ cross section and the SMD. Their validity has been carefully studied by $(e, 2 e)$ experiments on gas targets $[11,33,34]$, but further tests through studies of simple solid systems are essential.

Free electron systems, such as the alkali metals, would be ideal for initial (e,2e) studies of solids, but these materials are extremely reactive and difficult to handle. Aluminum is an excellent second choice. Hayes et al. studied a thin aluminum-aluminum oxide film, but did not observe the distinctive parabolic nearly-free-electron band in aluminum $[12,35,36]$. The observed spectra rather was dominated by $(e, 2 e)$ events from the surface oxide layers.

Though far more complex than the free electron metals, crystalline graphite has been studied extensively by various experimental techniques and by several theoretical methods [37]. The SMD of this material has been calculated using the LCAO approximation [38] and an $a b$ initio pseudopotential approach [15,39]. The two theoretical calculations are in semiquantitative agreement with each other and with an $(e, 2 e)$ experiment [15]. These studies provide some basis for analyzing systematic errors and for confirming the validity of the proportionality between the cross section and the SMD, but further studies on aluminum and graphite are desirable.

In parallel with studies of well understood solids have been several experiments to elucidate the conceptually challenging questions surrounding the electronic structure of amorphous carbon. Depending on the preparation conditions, this material can vary in properties from a black, soft semimetal to a hard, transparent insulator [40]. An obvious model for such a range of properties is 
based on the assumption that a variable mixture of the graphitic $\left(\mathrm{sp}^{2}\right)$ and diamond $\left(\mathrm{sp}^{3}\right)$ bonding (with possibly a small admixture of triple bonded sp form) exists in these materials. The SMD of these different bonds are quite distinctive and can be easily differentiated, in principle, by (e,2e) spectroscopy. As will be discussed in Section 4, the measured SMD of graphitic and diamondlike amorphous carbon are poorly modeled by a variable mixture of $\mathrm{sp}^{2}$ and $\mathrm{sp}^{3}$ bonding.

This review is organized as follows. In Section 2, the theory of (e,2e) spectroscopy, as it applies to solids, is discussed. The utility of various integrals of the SMD in analyzing experimental measurements is included in this section along with a discussion of multiple scattering. A description of different (e,2e) spectrometers is given in Section 3 along with a brief review of sample preparation methods. In Section 4 , a synopsis is presented of all $(e, 2 e)$ studies to date on the electronic structure of valence electrons in graphite, evaporated amorphous carbon, ion sputtered amorphous carbon, and aluminum-aluminum oxide films. Finally, a brief analysis of the design properties of an "ideal" (e,2e) spectrometer for studying solids is given in Section 5 .

\section{THEORY OF $(e, 2 e)$ SCATTERING}

\subsection{Kinematics}

An $(e, 2 e)$ reaction is a binary ionization event in which the kinematics of the two electrons is fully determined. Given the large scattering angles and high electron kinetic energies involved in experiments on solids, the colliding electrons can be treated like "billiard balls" as shown in Fig. 2. An electron with energy $E_{0}$ and momentum $\mathbf{P}_{\mathbf{0}}$, incident on a target, is scattered by a target electron into a final state of energy $E_{1}$ and momentum $P_{1}$. The recoiling target electron has energy and momentum $\mathrm{E}_{2}$ and $\mathbf{P}_{2}$, respectively. Conservation of energy and momentum give

$$
\begin{aligned}
& \mathrm{E}_{B}=\mathrm{E}_{\mathrm{o}}-\mathrm{E}_{1}-\mathrm{E}_{2}-\mathrm{E}_{\mathrm{r}} \\
& Q=\mathbf{P}_{\mathrm{o}}-\mathbf{P}_{1}-\mathbf{P}_{2}
\end{aligned}
$$

where the binding energy $E_{B}$ is the energy difference between the initial and ionized target state, $\mathbf{Q}$ is the recoil momentum of the target, and $\mathrm{E}_{\mathrm{r}}$ is the recoil energy. If $\mathrm{E}_{0} \gg \mathrm{E}_{B}$, and the mass of the 
target is much larger than the mass of an electron, then $E_{\mathbf{T}} \approx 0$ and $\mathbf{q}=-\mathbf{Q}$ where $\mathbf{q}$ is the momentum of the target electron prior to the collision.

The symmetric scattering geometry [Fig. 2a] in which $\theta_{1}=\theta_{2} \equiv \theta$ and $E_{1}=E_{2}$ [41] maximizes the momentum transferred to the recoiling electron and, thereby, assures that an important condition (see Section 2.3) for applying the impulse approximation to the calculation of the cross section is satisfied. Furthermore, if the incident electron kinetic energy is large, then both the scattered and recoiling electrons have high velocities and, therefore, long mean free paths so that multiple scattering (see Section 2.7) is minimized. In this geometry, the target electron momentum is

$$
\begin{aligned}
& \mathrm{q}_{\|}=2 \mathrm{P}_{1} \cos \theta-\mathrm{P}_{\mathrm{o}} \cos \phi \\
& \mathrm{q}_{\perp}=\mathrm{P}_{0} \sin \phi
\end{aligned}
$$

where $q_{\| l}$ and $q_{\perp}$ are parallel and perpendicular to the incident beam direction, respectively. The disadvantage of the symmetric scattering geometry is that the cross section for $(e, 2 e)$ scattering is a minimum when $\theta_{1}=\theta_{2} \approx 45^{\circ}$. In order to increase the count rate, Weigold et al. use an asymmetric scattering geometry [Fig. 2b] with $\theta_{1}=14^{\circ}$ and $\theta_{2}=76^{\circ}[24,25]$.

\subsection{Cross section}

The cross section for $(e, 2 e)$ scattering in the plane wave-impulse-independent electron approximation is (atomic units $\hbar=\mathrm{m}=\mathrm{e}=1$ )

$$
\frac{\mathrm{d} \sigma}{\mathrm{d} \Omega_{1} \mathrm{~d} \Omega_{2} \mathrm{dE}_{1} \mathrm{dE} E_{2}}=\frac{\mathrm{P}_{1} \mathrm{P}_{2}}{\mathrm{P}_{0}}\left(\frac{\mathrm{d} \sigma}{\mathrm{d} \Omega}\right)_{M o t t}\left|\mathrm{~F}\left(\mathbf{q}=\mathbf{P}_{1}+\mathbf{P}_{2}-\mathbf{P}_{0}\right)\right|^{2} \delta\left(\mathrm{E}_{\mathrm{B}}+\mathrm{E}_{0}-\mathrm{E}_{1}-\mathrm{E}_{2}\right)
$$

where $\left|F\left(\mathbf{q}=\mathbf{P}_{1}+\mathbf{P}_{2}-\mathbf{P}_{0}\right)\right|^{2}$ is the momentum density of the ground state orbital which was ionized by the binary collision and $\left(\frac{d \sigma}{d \Omega}\right)_{\text {Mott }}$ is the off-shell electron-electron Mott cross section [33]. A short discussion of the approximations in this expression and their validity is given in the next section. The crucial feature of $(e, 2 \mathrm{e})$ spectroscopy is that the cross section is proportional to the SMD; that is, the momentum density $|F(q)|^{2}$ of electron levels (in the independent electron approximation) with binding energies defined by the energy delta function in eqn. (5). If the 
momentum transfer $\mathbf{P}_{0}-\mathbf{P}_{1}$ is considerably larger than $\mathbf{q}$, then the proportionality constant between the cross section and the SMD is nearly independent of $q$ and $E_{B}$ [42]. The cross section for $(\mathrm{e}, 2 \mathrm{e})$ scattering is quite small because of the rapid drop off of the Mott cross section with momentum transfer $\left[\left(\frac{\mathrm{d} \sigma}{\mathrm{d} \Omega}\right)_{\text {Mott }} \propto\left[\mathbf{P}_{1}-\left.\mathbf{P}_{0}\right|^{-4}\right]\right.$ and because of the small fraction of available final states which are detected.

In planning experiments, it is useful to know roughly how the count rate will depend on experimental parameters such as incident energy, energy and momentum resolution, and electronic structure of the orbitals being studied. Using a simple model for the SMD, that it is constant for $\mathrm{E}_{B}=\mathrm{E}(\mathbf{q})$ and $\mathrm{q} \leq \mathrm{q}_{c}$ and zero otherwise (this might be an S-like band which does not cross the Fermi surface), Amaldi and Atti [43] have shown that the count rate can be written

$$
\mathrm{I}_{(\mathrm{e}, 2 \mathrm{e})} \propto \frac{\eta \mathrm{tI}_{0} \mathrm{q}_{c}}{\mathrm{E}_{0}^{7 / 2}}\left(\frac{\Delta \mathrm{q}}{\mathrm{q}_{c}}\right)^{4} \Delta \mathrm{E}
$$

where $\eta$ is the density of primitive cells in a crystal (the density of atoms in amorphous solids), $t$ is the thickness, $\mathrm{I}_{0}$ is the incident current, $\mathrm{E}_{0}$ is the incident energy, and $\Delta \mathrm{q}$ and $\Delta \mathrm{E}$ are the momentum and energy resolution, respectively [see Appendix for the derivation of eqn. (6)]. The strong dependence of $\mathrm{I}_{(\mathrm{e}, 2 \mathrm{e})}$ on $\Delta \mathrm{q} / \mathrm{q}_{\mathrm{c}}$ demonstrates the value of having adjustable momentum resolution [14]. In practice, the count rate is heavily influenced by multiple scattering which is not taken into account in eqn. (6); this complication is discussed in Section 2.7.

\subsection{Justification of approximations}

A derivation of the $(\mathrm{e}, 2 \mathrm{e})$ cross section is quite complex since it is at best a 3-body problem for hydrogen and at worst a many-body problem for solids. Equation (5) was obtained assuming the plane-wave impulse (PWIA) and independent-electron approximations. The most compelling evidence for the validity of these approximations is the excellent agreement between theory and experiment for a large number of atomic and molecular systems $[11,33,34,44]$. Initial evidence 
validating the application of eqn. (5) to solid targets comes from experimental and theoretical studies of crystalline graphite [15,35].

The PWIA is generally valid for solid targets because of the high incident energy and large momentum transfer used to minimize multiple scattering. For the impulse approximation to be valid, the collision must be localized and must occur on a time scale which is shorter than the response time of the target system. The length scale for the volume in which the collision is localized is set by the inverse of the momentum transfer. In the symmetric scattering geometry, this scale is $0.02 \AA$ ( $E_{0}=25 \mathrm{keV}$ ) which is much less than the average separation of electrons in solids. The fastest time scale for the electronic system to relax is the period of the plasma oscillation, which in solids is $\sim 10^{-16} \mathrm{~s}$. This time period is four orders of magnitude longer than the time required for the scattered and recoiling electrons $(E=12.5 \mathrm{keV})$ to leave a typical unit cell. McCarthy and Weigold review the PWIA for atomic and molecular systems and conclude that in the symmetric scattering geometry, it is valid for $E_{0}>1200 \mathrm{eV}$ [33] providing further justification for the validity of this approximation in (e,2e) experiments on solids.

The independent electron approximation is valid in solids when the electron-electron correlation energy is small, as in valence bands derived from $\mathrm{s}$ and $\mathrm{p}$ atomic orbitals. Where electron-electron correlation effects are large, such as in core levels and valence bands derived from $\mathrm{d}$ and $\mathrm{f}$ atomic orbitals, the approximation is expected to break down. The signature for the breakdown of this approximation, which is observed in atomic and molecular systems, is the appearance of satellite structures in the (e,2e) spectrum $[11,33,34,44]$. To date, all $(e, 2 e)$ studies on solids have been on broad, weakly-correlated valence bands and no satellite structure has been observed.

For completeness we mention other approximations incorporated in the cross section for (e,2e) scattering from solids which are quite rigorously justified. As discussed earlier, the symmetric scattering geometry and high incident energies assure the interaction volume is quite localized and that one can neglect diffraction effects arising from the wave nature of the electron. The effects of electron diffraction have been studied theoretically [45-47] and may be significant in 
an asymmetric scattering geometry where the momentum transfer is small $[25,48]$. The influence of relativistic effects is $<5 \%$ for all present spectrometers which use $E_{0}<50 \mathrm{keV}$. Both the recoil energy of the ionized ion and the temperature smearing of the Fermi surface can be neglected given that both are significantly less than the energy resolution of all existing spectrometers.

\section{$2.4 \quad$ Spectral momentum density of crystalline solids}

The wavefunction of an orbital in a crystalline solid in the independent electron approximation can be written by Bloch's theorem as

$$
\Psi_{\mathbf{n}, \mathbf{k}}(\mathbf{r})=\mathrm{e}^{\mathbf{i} \mathbf{k} \cdot \mathbf{r}} \mathbf{u}_{\mathbf{n}, \mathbf{k}}(\mathbf{r})
$$

where $\mathbf{n}$ is the band index, $\mathbf{k}$ is the crystal-momentum and lies within the Brillouin zone, and $\mathrm{u}_{n, k}(\mathbf{r})$ has the translation symmetry of the crystal. The SMD of this orbital is

$$
\left|F_{\mathbf{n}, \mathbf{k}}(\mathbf{q})\right|^{2}=\sum_{\mathbf{G}} \delta_{\mathbf{q}, \mathbf{k}+\mathbf{G}}\left|\mathrm{u}_{\mathbf{n}, \mathbf{k}, \mathbf{G}}\right|^{2} \delta\left(\mathrm{E}-\mathrm{E}_{\mathbf{n}}(\mathbf{k})\right)
$$

where $\mathbf{G}$ is a reciprocal lattice vector and $u_{n, k, \mathbf{G}}$ is the reciprocal lattice expansion coefficient of $\mathrm{u}_{\mathbf{n}, \mathbf{k}}(\mathbf{r})$. The real momentum $\mathbf{q}$ is not restricted to the Brillouin zone. This general expression for the SMD provides minimal insight into the electronic structure of crystalline solids. However, it is useful to consider the two limiting approximations introduced in Section 1 and shown schematically in Fig. 1 which correspond to large (nearly-free) and small (tight-binding) overlap of the neighbor atomic wave functions. These limits were discussed previously by Williams and Hayes [12].

In the limit of nearly free electron, $u_{n}, \mathbf{k}, \mathbf{G}$ is constant and $E_{n}(\mathbf{q})$ disperses quadratically with momentum except near a Brillouin zone boundary where a gap opens in the energy dispersion and $\mathrm{u}_{\mathbf{n}, \mathbf{k}, \mathbf{G}}$ drops rapidly to zero passing from one Brillouin zone to the next (repeated zone scheme) $[2,13 ; 49]$. The next band above the gap continues the free electron parabolic energy dispersion until it approaches the next Brillouin zone boundary. If the Fermi energy lies within a band, then the SMD will be cut off sharply by the Fermi function. 
In the opposite tight-binding limit for a band derived from nondegenerate atomic orbitals, one obtains $u_{n, k}, \mathbf{G}=\emptyset_{n, k}(\mathbf{q}=\mathbf{k}+\mathbf{G})$ where $\emptyset_{n, \mathbf{k}}(\mathbf{q})$ is the momentum transform of the atomic orbital. An extreme case of a core $1 \mathrm{~s}$ level also is shown in Fig. 1. Since the atomic core orbitals are tightly bound in real space, the SMD for the band will extend out through several Brillouin zones in sharp contrast to the nearly-free electron limit where the SMD for a given band is strongly localized to one Brillouin zone.

\subsection{Spectral momentum density of amorphous solids}

With the loss of translational symmetry in disordered solids, the crystal momentum $\mathbf{k}$ is no longer a good quantum number and, therefore, the band dispersion relation $\mathrm{E}(\mathbf{k})$ no longer has meaning. But the SMD is well defined and given in the independent electron approximation by

$$
\rho(E, \mathbf{q})=\sum_{\alpha}\left|\Phi_{\alpha}(\mathbf{q})\right|^{2} \delta\left(E-E_{\alpha}\right)
$$

where $\alpha$ labels the eigenstates of the independent electron Hamiltonian and $\Phi_{\alpha}(\mathbf{q})$ is the one electron orbital in the momentum representation. The difficulty, of course, is in finding the eigenvalues and eigenfunctions of the Hamiltonian when one is unable to factorize the secular determinant because of the absence of symmetry operators which commute with the Hamiltonian. In spite of this daunting hurdle, there have been several theoretical attempts to calculate the SMD of amorphous solids. They have been based primarily on the continuous-random-network model for tetrahedrally bonded solids [50]. These studies find remarkably well-defined energy-momentum correlations in the amorphous solid which are similar to an angular average of the $E$ vs. $\mathbf{k}$ band dispersion in the crystalline counterpart.

One theoretical approach to finding the eigenstates and eigenfunctions of a disordered system is to take advantage of approximate symmetries, such as the short-range order, which exist in many of these systems. Sadoc and Mosseri, for example, developed the elegant idea that an approximate symmetry in real space might be an exact symmetry in curved space [51]. Di Vincenzo

et al. applied this concept to amorphous tetrahedreally bonded semiconductors [52]. Specifically, 
they argued that some long-range order persists in these materials due to the constraints imposed by the existence of short-range order and that states generated by the exact symmetry in curved space can provide a good basis set for representing the eigenstates in real space. Haydock also developed a theoretical procedure for generating eigenstates of a covalently bonded, disordered semiconductor based on the long-range symmetry arising from short-range order [53]. Gibson and Haydock found an effective medium Hamiltonian based on a network momentum operator for a three-fold coordinated continuous random network structural model [54]. From their model they calculated the SMD of graphitic amorphous carbon. Beeby and Hayes [55] used an alternate method based on a Green's function technique. They calculated the electronic structure at a given site based on the local short-range order taking into account the positionally disordered distant neighbor sites in an iterative, self-consistent fashion.

A second approach to calculating the SMD of disordered solids is based on determining the electronic structure of a finite size cell in which the positions of the atoms are random to the maximum extent allowed by local bonding constraints. Hickey and Morgan [56], using cells of tetrahedrally bonded networks [57], calculated the SMD using an equation of motion technique with. a plane wave basis set and with the local electronic structure modeled by a pseudopotential. Bose et al. used the same cells and a similar recursion technique [58]. Both works found, again, strong energy-momentum correlations in the SMD similar to the angular averaged crystalline band dispersion.

The only direct measurement of the SMD of an amorphous solid has been in studies of amorphous carbon $[3,35,38]$ by (e,2e) spectroscopy where energy-momentum correlations similar to the band structure of crystalline graphite $[15,35]$ have been observed.[59] [There are also significant differences. See Sections 3.3.1 and 3.3.2]. Though direct comparison between theory and these experiments is difficult because carbon forms both trigonal $\left(\mathrm{sp}^{2}\right)$ and tetrahedral $\left(\mathrm{sp}^{3}\right)$ bonds with its neighbors, the evidence is strong that vestiges of crystal band dispersion remain in amorphous solids. Thus, (e,2e) spectroscopy is beginning to address the basic question raised by Welber and Brodsky almost twenty years ago: "For those amorphous materials that are characterized by an 
absence of long-range order and a retention of short-range order. . what remains, if any, of the crystal band structure...." [60].

\subsection{Integrals of the spectral momentum density}

The SMD $\rho(E, q)$ obtained from the $(e, 2 e)$ spectrum can be integrated to obtain the energy density of states $N(E)$ and the momentum density $\mathrm{J}(\mathbf{q})$

$$
\begin{aligned}
& N(\mathrm{E})=\int \mathrm{d} \mathbf{q} \rho(\mathrm{E}, \mathbf{q}) \\
& \mathrm{J}(\mathbf{q})=\int \mathrm{dE} \rho(\mathrm{E}, \mathbf{q})
\end{aligned}
$$

where integral (10) can be compared to angle-integrated photoemission spectra and integral (11) can be compared to Compton scattering and positron annihilation measurements. These integrals provide useful checks on the (e,2e) data though eqn. 10 can be applied to data from measurements on single crystals only if a large fraction (fraction depends on crystal symmetry) of momentum phase space is studied. It is generally not feasible to cover such a large fraction of phase space because of the low $(e, 2 e)$ count rate. In the case of amorphous (and polycrystalline) solids, the SMD is rotationally invariant so that integral (10) reduces to a one-dimensional integral and profitably can be compared to photoemission measurements.

An important integral which can, in principle, allow one to determine the absolute spectral momentum density is

$$
\mathrm{n}=\int \mathrm{dE} \int \mathrm{d} \mathbf{q} \rho(\mathrm{E}, \mathbf{q})
$$

where $n$ is the total density of electrons in the solid. Generally the binding energies of the valence and core electrons are well separated so that one can write

$$
\mathrm{n}_{\text {valence }}=\int_{\text {valence }} \mathrm{dE} \int \mathrm{d} \mathbf{q} \rho(\mathrm{E}, \mathbf{q})
$$

where $n_{\text {valence }}$ is the density of valence electrons. Again, integral (13) is not helpful for crystalline solids as data cannot be obtained over a sufficiently large volume of momentum space.

A useful extension of integral (13) for studying amorphous solids is the partial integral 


$$
\mathrm{n}_{\text {valence }}(\mathrm{E})=\int_{0 \text { valence }}^{\mathrm{E}} \mathrm{d} \mathrm{E}^{\prime} \int \mathrm{d} \mathbf{q} \rho\left(\mathrm{E}^{\prime}, \mathbf{q}\right)
$$

where zero energy generally is the Fermi level. The utility of this partial integral is that one may have some information regarding the atomic or molecular valence band levels of the constituents making up the amorphous solid. The question then is; how do these atomic and molecular levels shift, broaden, and hybridize in the solid. By comparing the measured fraction $\mathrm{n}_{\text {valence }}(\mathrm{E}) / \mathrm{n}_{\mathrm{valence}}$ as a function of $E$ to the fraction calculated from the atomic and molecular levels, one can obtain insight into the nature of the electronic levels in the solid. In practice, one compares $n_{\text {valence }}(E, q)$ to theory where

$$
n_{\text {valence }}(E, \mathbf{q})=\int_{0}^{E} d E^{\prime} \int_{o}^{q} \mathbf{q}^{\prime 2} d \mathbf{q}^{\prime} p\left(E^{\prime}, \mathbf{q}^{\prime}\right)
$$

for disordered solids. This is necessary because the experimental SMD often is lost in noise at moderate values of $\mathbf{q}$ well before the momentum integral has converged to the $q \rightarrow \infty$ limit.

\subsection{Implications of multiple scattering for solids}

The application of $(\mathrm{e}, 2 \mathrm{e})$ scattering to solids revealed contributions to the spectrum from multiple scattering events, a new feature not previously seen in $(e, 2 e)$ measurements on gas targets. The effect of multiple scattering can be seen (Fig. 3) in the extended data of Kheifets et al. from evaporated amorphous carbon [38] and Hayes et al. from aluminum-aluminum oxide films [36] which show the coincidence rate as a function of binding energy (with respect to the Fermi energy) for momentum $q=0$. In the absence of multiple scattering, the spectrum in the independent electron approximation would be zero between the bottom of the valence band, $21 \mathrm{eV}$ for amorphous carbon and $\sim 28 \mathrm{eV}$ for aluminum oxide, and the first core level. Instead, as seen in the figures, a nearly constant spectrum extends below the bottom of the valence band to the limits of the measurements. This continuum below the valence band is due to multiple scattering events consisting of a binary collision plus one or more small angle scattering events before or after the 
(e,2e) collision. The small angle collisions are predominantly from the creation of plasmons. An analysis of multiple scattering contributions to $(e, 2 e)$ spectra is given by Jones and Ritter [62]. Hayes et al. $[12,35,36]$ used an alternative Monte Carlo deconvolution procedure to correct for multiple scattering in their data.

The relatively high probability for small angle scattering events in solids has an enormous impact on the optimum target thickness for an $(e, 2 e)$ experiment on a given material. The (e,2e) rate is obtained from coincidence timing spectrum [see Fig. 4(a)] which ideally consists of a large coincidence peak from true events of interest riding on a small background from uncorrelated scattering events in which two electrons by chance arrive at the two detectors within the coincidence timing window $\Delta \mathrm{t}_{\mathrm{c}}[11,33,34]$. In brief, multiple scattering reduces the true signal rate without substantially affecting the background rate so that with increasing target thickness, the ratio of the signal to background count rates falls off exponentially with the thickness/mean free path ratio. In the limit of zero target thickness, the signal/background ratio diverges but the signal rate goes to zero. So, there is an optimum target thickness which depends on the mean free path for small angle scattering. The mean free path is approximately proportional to the energy of the electron, so the optimum target thickness depends sensitively on the incident electron energy.

The optimum target thickness can be obtained from the expression derived by Lower and Weigold [19] for the time T required to obtain a given statistical uncertainty $\delta$ in the total count taking into account the deleterious effect of multiple scattering

$$
\mathrm{T} \propto \frac{1}{\delta^{2} \mathrm{f} \triangle_{0} \mathrm{I}_{\mathrm{o}}}
$$

where $\delta=\Delta \mathrm{N}_{\mathrm{t}} / \mathrm{N}_{\mathrm{t}}\left(\Delta \mathrm{N}_{\mathrm{t}}\right.$ is the statistical variance in the number of true coincidence events and $\mathrm{N}_{\mathrm{t}}$ is the number of true coincidence events), $I_{0}$ is the incident beam current, $\Delta_{0}$ is the thickness of the sample, and $\mathrm{f}$ is the fraction of single scattering events. (This factor $\mathrm{f}$ was not present in the original expression of Lower and Weigold). Jones and Ritter [61] find for the symmetric (e,2e) geometry

$$
\mathrm{f}=\frac{\Lambda\left(\mathrm{E}_{0}\right) / \Delta_{0}}{4 \sqrt{2}-1}\left\{\exp \left[-\Delta_{0} / \Lambda\left(\mathrm{E}_{\mathrm{o}}\right)\right]-\exp \left[-4 \sqrt{2} \Delta_{0} / \Lambda\left(\mathrm{E}_{0}\right)\right]\right\}
$$


where $E_{0}$ is the incident energy and $\Delta\left(E_{0}\right)$ is the mean free path of the incident electron. For $E_{0} \geq 1 \mathrm{keV}, \Lambda\left(E_{0}\right)$ is proportional to $E_{0} 1 n\left(E_{0}\right)$. In the limit $\Lambda\left(E_{0}\right) \gg \Delta_{0}$, one can see that $f \rightarrow 1$.

In order to minimize the data collection time $\mathrm{T}$ for a given relative statistical uncertainty $\delta$, one must maximize the incident current $\mathrm{I}_{O}$ and the effective thickness of the target $f \Delta_{0}$. In practice, the incident beam current is limited by sample heating and the Boersch effect (rapidly deteriorating energy resolution with increasing incident beam current) [62,63]. The maximum value of $f \Delta_{0}$ as a function of $\Delta_{0} / \Lambda\left(E_{0}\right)$ occurs when the target thickness is $\Delta_{0}=0.37 \Lambda\left(E_{0}\right)$. At this optimum thickness, $33 \%$ of the integrated $(\mathrm{e}, 2 \mathrm{e})$ spectrum is single scattering binary events of interest. Thus, at the optimum thickness the spectrum still is significantly influenced by multiple scattering. One must either correct for the multiple scattering by some deconvolution method or use thinner samples at the cost of longer data accumulation times. One can also increase the incident energy $\mathrm{E}_{0}$ to increase $\Lambda\left(E_{0}\right)$ and, thereby, reduce the multiple scattering, but at a stiff price; the data accumulation time $T$ increases proportional to $\mathrm{E}_{0}{ }^{3}$ in the limit $\Delta_{0} / \Lambda\left(\mathrm{E}_{\mathrm{O}}\right) \ll<$.

Jones and Ritter demonstrated that the observed $(e ; 2 \mathrm{e})$ spectrum is proportional to the convolution of a "smearing function" $S(E, q)$, which accounts for all kinematically allowed small angle scattering, with the $(\mathrm{e}, 2 \mathrm{e})$ cross section of interest [64]. The smearing function depends explicitly on energy and momentum and implicitly on the parameters $\Delta_{0} / \Lambda\left(\mathrm{E}_{0}\right)_{\text {inelastic }}$ and $\Delta_{0} / \Lambda\left(\mathrm{E}_{\mathrm{o}}\right)_{\text {elastic }}$ where $\Lambda\left(\mathrm{E}_{\mathrm{o}}\right)_{\text {inelastic }}$ and $\Lambda\left(\mathrm{E}_{\mathrm{O}}\right)_{\text {elastic }}$ are the inelastic and elastic mean free paths, respectively. The target thickness and mean free paths often are poorly known. One can estimate these parameters and obtain a quick characterization of the degree of multiple scattering in a sample

from the singles spectrum taken by one analyzer of the (e,2e) spectrometer [64]. An example of such a spectrum for amorphous carbon is shown in Fig. 4(b).

\section{EXPERIMENT}

\subsection{Spectrometers}

Schematics of two $(\mathrm{e}, 2 \mathrm{e})$ spectrometers $[14,19]$ are shown in Fig. 5. They are representative of other instruments for solid targets $[4,13,18,24-26,41]$. The three primary elements are the electron 
source, target chamber/sample, and energy and momentum analyzers/electron multipliers. For the symmetric scattering geometry, it is convenient to have the energy analyzers/electron multipliers near ground potential which requires that the electron source and target chamber be at respective potentials $\mathrm{V}_{G}<0$ and $\mathrm{V}_{T}>0$. Those coincidence scattering events are detected in which the incident and recoiling electrons have equal kinetic energies. If the pass energy of the analyzers is $E_{p}$ and the target electron binding energy with respect to the Fermi energy is $-\mathrm{E}_{B}\left(\mathrm{E}_{\mathrm{B}}>0\right)$, then

$$
\mathrm{E}_{B}=-\mathrm{eV}_{G}-\mathrm{eV}_{T}+\mathrm{W}_{C}+2 \mathrm{E}_{p}-2 \mathrm{~W}_{A}
$$

where $\mathrm{W}_{C}$ and $\mathrm{W}_{A}$ are the work functions of the electron source cathode and the analyzer, respectively. High quality commercial voltage supplies have a long-term stability of about $50 \mathrm{ppm}$. When $\left|V_{G}\right|$ and $V_{T}$ are greater than $10 \mathrm{keV}$, then long-term drift in these supplies is a significant source of uncertainty in determining $\mathrm{E}_{\mathrm{B}}$. The certainty in $\mathrm{W}_{C}$ and $\mathrm{W}_{A}$ (known to $\sim \mathrm{leV}$ ) and charging of insulating samples introduce further systematic errors. Ideally, zero binding energy would be calibrated by observing the distinctive cut-off of the Fermi function in a metal, as is done in photoemission spectroscopy, but this cut-off has not yet been observed in (e,2e) spectra.

The electron source generally is a commercial triode electron gun. The energy spread of the electron beam is thermal for low currents and increases with current above $\sim 1 \mu \mathrm{A}$ due to the Boersch effect [62,63]. One can reduce the energy spread of the electron source below the thermal width $(\sim 0.25 \mathrm{eV})$ with a monochromator, but there is significant loss in current with increasing resolution. Kuyatt and Simpson made a careful study of the hemispherical analyzer as a monochromator optimizing the beam optics through it for maximum energy resolution and current [65]. They obtained $0.1 \mu \mathrm{A}$ of current with a spread of $0.1 \mathrm{eV}$ and found the current scaled as $\sim \Delta \mathrm{E}^{5 / 2}$. Therefore, improvement of the energy resolution in $(e, 2 \mathrm{e})$ spectroscopy below the thermal spread will require substantial implementation of parallel detection techniques to maintain a reasonable data rate.

The energy analyzers in the spectrometer shown in Fig. 5(a) were Wien filters [crossed $\mathbf{E}$ and B fields] with resolutions of $\sim 4 \mathrm{eV}$ FWHM [14]. For high resolution, hemispherical analyzers [see Fig. 5(b)] have a very high figure of merit [66], significantly better than the Wien filter, and the 
hemispherical analyzer also couples naturally to microchannel plate detectors and position sensitive detectors for parallel detection of a range of energy and momentum[18,19,23-26]. For an asymmetric scattering geometry and parallel collection of data, Storer et al. [24,25] combined a hemispherical and a toroidal analyzer in their spectrometer to obtain optimum coupling of the scattered beams to the energy analyzers. The fastelectron (scattered in the forward direction) and the slow electron (the large scattering angle) were focused into the hemispherical and toroidal analyzers, respectively.

The target electron momentum is determined by the momenta of the incident, scattered, and recoiling electrons (refer to Eqs. 3 and 4 and Fig. 2). For spectrometers similar to the one shown in Fig. 5(a), the component of target electron momentum perpendicular to the incident beam is chosen by varying the angle $\phi$ of the incident beam with pairs of electrostatic deflectors which rotate (without translation) the direction of the incident beam on the target. The component of target electron momentum parallel to the incident beam is chosen by sets of electrostatic deflectors in the exit paths of the scattered and ejected electrons which symmetrically rotate the opening angle $\theta$ of these two beams. The scale of momentum one would like to study is typically set by the Brillouin zone boundary which is of order $2 \AA^{-1}$. The change in momentum with respect to angle is $0.08 \AA^{-1} / \mathrm{mrad}$ for an incident energy of $25 \mathrm{keV}$ so that an angular range of order $\pm 40 \mathrm{mrad}$ must be covered with an angular precision approaching a few milliradians. This level of precision places severe demands on the alignment and calibration of the electrostatic deflectors.

The deflectors in front of the target can be calibrated by observing the Bragg diffraction peaks of a suitable target, such as polycrystalline aluminum, with an energy analyzer and detector placed behind the target. The electrostatic deflectors behind the target also can be calibrated by Bragg scattering if electron sources are placed in front of the target in line with the detectors for the scattered and ejected electrons. In practice, Ritter et al. [14] scaled the calibration of the deflectors behind the target to the calibration of the deflectors in front of the target by the relative kinetic energies of the incident and scattered electrons and by the appropriate relative dimensions of the two groups of deflectors. The determination of the absolute momentum is based on suitably placed 
apertures to define the electron optics of the incident and scattered beams. In practice, mechanical misalignment of these apertures and uncertainty regarding the position of the electron beam on the target has been a major source of systematic error in determining the momentum [14].

\subsection{Electronics and signal processing}

The first (e,2e) spectrometers applied to solids (stages one and two in our historical sequence) collected data for the energy-momentum phase space of interest in serial order. The energy is determined from the energy of the incident, scattered, and ejected electrons while the momentum is reconstructed from the scattering geometry. The two detected electrons can be scattered and ejected electrons from binary vertices of interest or scattered electrons from two separate binary events which are uncorrelated in time. These true and accidental scattering events are disentangled by coincidence spectroscopy. One electron starts a time-to-amplitude converter (or time-to-digital converter) while the second detected electron stops the converter (with an arbitrary delay $t_{0}$ so that events of interest do not appear at the electronically ill-defined zero time). The true events appear as a peak centered at $t_{0}$ riding on the uncorrelated accidental events which appear as background spread uniformly across the time spectrum [Fig. 4(a)]. The true plus accidental count is determined from integrating the spectrum over a time window $\Delta t_{c}$ centered at $t_{o}$ while the accidental count is measured from the background spectrum in a time window $\Delta \mathrm{t}_{\mathrm{b}}$ well separated from the delay time. The true events are then obtained by subtracting the background count from the true plus accidental count taking into account the relative widths of the two windows.

The parallel collection of data (stage three in our historical sequence) is done with microchannel plates and resistive anode position sensitive detectors on the output focal plane of an electrostatic analyzer. The first parallel-collection spectrometers used only one dimension of the position sensitive detector to collect a range of the energy spectrum [13,18,19,26]. Recently completed spectrometers use both dimensions of the position sensitive detector to obtain energy and momentum information in parallel [23-25,28]. The rate at which information must be processed 
to obtain the true coincidence rate as a function of energy and momentum expands significantly in a parallel detection system. The position of the detected electrons on the resistive anode strip must be translated into the binding energy and momentum and the timing information must be corrected for the dispersion in transit times of electrons passing through the hemispherical analyzers with different energies. This dispersion contributes to the width of the true coincidence peak and seriously degrades the signal to background ratio. Techniques have been developed to compensate for this effect $[19,27]$.

Two approaches address the expanded information processing in parallel data collection. The Flinders group relies heavily on NIM hardware both for translating the resistive anode signal into an energy-momentum value and for correcting the timing signal [13,18,24,25]. A computer performs higher level functions such as stepping energy and/or momentum ranges, storing the coincidence and background counts as a function of energy and momentum, and providing graphical displays, The second approach, taken by the group at Perth, relies more on software to translate lower level resistive anode and timing signals into higher level information [23,26]. This approach relies less on analog signal processing and permits tuning the experiment by computer.

\section{3:3. Sample preparation}

Each material to be studied by $(e, 2 e)$ spectroscopy presents a new, formidable challenge for sample preparation because of the extremely thin samples which are required $(\sim 100 \AA$ for incident beam energies $\sim 25 \mathrm{keV}$ ) in order to minimize multiple scattering. As discussed in Section 2.7 , the optimum sample thickness is $37 \%$ of the mean free path of the incident electron. The elastic and inelastic mean free paths can be estimated from

$$
\begin{aligned}
& \Lambda_{\text {elastic }}(\mathrm{E})=882 \AA(\mathrm{E} / 25 \mathrm{keV}) \frac{\mathrm{A}}{\rho \mathrm{Z}^{4 / 3}} \\
& \Lambda_{\text {inelastic }}(\mathrm{E})=48 \AA(\mathrm{E} / 25 \mathrm{keV}) \frac{\mathrm{A}}{\rho \mathrm{Z}^{1 / 3}}
\end{aligned}
$$


using expressions given by Crewe where $\mathrm{A}$ is the atomic weight, $\mathrm{Z}$ is the atomic number, and $\rho$ is the specific gravity [67]. The total mean free path is dominated by the inelastic contribution and varies from $150 \AA$ for carbon to $900 \AA$ for cesium when $E_{0}=25 \mathrm{keV}$.

Well developed techniques exist for preparing samples for transmission electron microscopy [68]. These foils can be of order or greater than $1000 \AA$ thick since $E_{0} \geq 100 \mathrm{keV}$ for TEM. The area of a thin foil only need be of order a few square microns since the beam is strongly converging at the target. For (e,2e) spectroscopy, the film must be thinner by nearly an order of magnitude and the area of the foil must be six orders of magnitude larger for a momentum resolution of $0.1 \AA^{-1}$ (at $E_{0}=25 \mathrm{keV}$ ). High-energy, nonimaging, transmission EELS spectrometers operate at the same energies as TEM, requiring, therefore, films of the same thickness. But samples for EELS must be of comparable area as $(e, 2 e)$ samples because both techniques have similar momentum resolution. Some techniques for preparing transmission EELS samples have been discussed by Fields and Fink [69].

Amorphous and polycrystalline films often can be prepared by evaporation or sputtering of the material either on detergent-coated glass slides or on rock salt crystals. They are floated off in water and mounted either across an aperture or on a TEM grid. A difficulty with many of these films is the oxide surface layer which can be comparable in thickness to the film itself. Preparing good quality, single-crystal films which are several thousand angstroms thick is difficult: going another order of magnitude thinner is heroic. One general approach which can be applied to many materials is to prepare an $1000 \AA$ thick foil using standard techniques from EELS and TEM and, then, approach the final thickness using ion milling or reactive ion etching. There is evidence that plasma etching does less damage to the surface than ion milling [70].

\section{EXPERIMENTAL STUDIES OF SOLIDS}

Four systems have been studied with sufficient resolution to observe distinct features in the SMD of the valence band. Several groups have studied evaporated amorphous carbon which has 
become the operational standard for evaluating a new spectrometer. Graphite and diamondlike amorphous carbon have been studied by the group at Virginia Tech and graphite and an aluminumaluminum oxide film has been investigated by the group at Perth. We will discuss graphite first because the theoretical SMD can be calculated quite accurately for this material and because the experimental SMD of amorphous carbon and crystalline graphite are remarkably similar. A theoretical framework for calculating the SMD of amorphous solids is much less well developed than the corresponding theory of crystalline solids and comparison of the crystalline and disordered spectra may provide guidance in developing such a framework.

\subsection{Graphite}

The $(\mathrm{e}, 2 \mathrm{e})$ spectra of partially oriented graphite was measured by Gao et al. for momentum parallel and perpendicular to the c-axis and the results were compared to a first principles calculation of the SMD [15]. A relatively thick film was prepared by the "standard scotch-tape" method from a crystalline graphite inclusion in quartz. This film, mounted across an aperture, was thinned further by reactive ion etching using an oxygen plasma. The c-axis of the crystal was perpendicular to the film, but the domain structure of the basal plane was not determined. All evidence from Raman spectroscopy and electron diffraction suggested that the crystalline order of the original graphite flake survived the trauma of the thinning process [15,71].

Graphite has a simple hexagonal Bravais lattice with a basis of four atoms. The reciprocal lattice is shown in Fig. 6(a). The band structure of graphite, calculated by Holzwarth et al., is also shown in Fig. 6(a) [39]. They used a first-principles, self-consistent technique based on densityfunctional theory in the local-density approximation using the mixed-basis pseudopotential approach. The sixteen valence electrons per primitive cell fill eight bands consisting of three $\sigma$ doublets and one $\pi$ doublet. The $\sigma$ doublets are nearly degenerate because of the weak interaction between $\mathrm{sp}^{2}$ orbitals in the neighboring hexagonal sheets, while the two $\pi$ bands are split by nearly $2 \mathrm{eV}$ at the $\Gamma$ point because of the relatively large overlap of the $p_{z}$-like orbitals on adjacent sheets. 
The band structure of graphite has been measured by angle-resolved photoemission and excellent agreement exists between the experimental results [72] and the calculations of Holzwarth et al.

The SMD is directly related to the expansion coefficients of the Bloch wave functions [see eqn. (8)]. These coefficients were calculated by Gao et al. [15] by the same theoretical approach as was used by Holzwarth et al. in calculating the band structure and are in fair agreement with a similar calculation by Kheifets et al. [38]. The SMD is plotted in Fig. 6(b-d) in the high symmetry directions $\mathrm{q}=\Gamma \rightarrow \mathrm{A}, \mathrm{q}=\Gamma \rightarrow \mathrm{K}$, and $\mathrm{q}=\Gamma \rightarrow \mathrm{M}$ for the three $\sigma$ and one $\pi$ doublets. The momentum densities of the two members of the doublets are nearly the same in all four cases. The different partial wave symmetries of the bands are clearly evident. The S-like character of the $\sigma_{1}$ bands is seen from the monotonic fall-off of the SMD with increasing momentum both perpendicular and parallel to the c-axis. The SMD of the P-like $\sigma_{2}$ and $\sigma_{3}$ bands are zero along the c-axis. In the $\Gamma \rightarrow \mathrm{K}$ and $\Gamma \rightarrow \mathrm{M}$ directions, the SMD of the $\sigma_{2}$ bands are negligible out to $\sim 1.0 \AA^{-1}$ and then go through a peak just outside the first Brillouin zone. The momentum densities of the $\sigma_{3}$ bands in these directions are negligible until well into the second Brillouin zone. The momentum densities of the $\pi$-bands have a node in the basal plane and go through a broad maximum in the second and third Brillouin zones.

The experimental measurements by Gao et al. of the SMD of the graphite bands as a function of energy for different momenta are shown in Fig. 7 [15]. In Fig. 7(a), the momenta, in steps of $0.6 \AA^{-1}$ perpendicular to the c-axis, include a constant component $q_{\| \mid}=-0.61 \AA^{-1}$ parallel to the caxis due to a misalignment of the spectrometer. In Fig. 7(b), the momenta, in steps of $0.6 \AA^{-1}$ parallel to the $c$-axis, include a constant component $q_{\perp}=-0.35 \AA^{-1}$ perpendicular to the c-axis. The energy resolution for these measurements was $8.6 \mathrm{eV}$ FWHM and the momentum resolution was estimated to be $0.47 \AA^{-1}$ FWHM and $0.73 \AA^{-1}$ FWHM for momentum parallel and perpendicular to the $c$-axis, respectively. Statistical error bars are shown for each spectra.

The theoretical SMD are shown as bars in Fig. 7. The widths of the bars are equal to the splitting of the respective band doublets. The calculations were made for the actual measured momentum, taking into account the misalignment of the spectrometer, so that a weak peak is 
predicted for the $\pi$-band in Fig. 7(a) even though the nominal momentum is perpendicular to the caxis. For momentum in the basal plane, the theoretical results for the $\Gamma \rightarrow M$ direction are plotted in Fig. 7(b) since variations in the theoretical SMD between the $\Gamma \rightarrow M$ and $\Gamma \rightarrow K$ directions are smaller than the scatter in the experimental data. The experimental data has been scaled to the theoretical results using the peak at $20 \mathrm{eV}$ in the $\mathrm{q}=0.0 \AA^{-1}$ spectra in Fig. 7(a).

One can clearly see in Fig. 7(a) the dispersion of the $\sigma$ bands for momentum in the basal plane despite considerable scatter in the data. The experimental dispersion appears to be stronger than the theoretical prediction, but the experimental and theoretical SMD are in good agreement for these bands. For momentum parallel to the c-axis, the $\sigma$ and $\pi$ bands are predicted to be nearly dispersionless and this is confirmed by the data in Fig. 7(b). The P-wave behavior of the $\pi$ band is also clearly evident with the SMD going through a maximum at q $2 \AA^{-1}$. A stringent test of the theory is the predicted ratio of the maximum SMD in the $\pi$ and $\sigma$ bands. The observed ratio is, in fact, in good agreement with the theoretical prediction.

\subsection{Amorphous carbon}

The properties of amorphous carbon films vary, depending on how they are prepared and on how much hydrogen is incorporated into the material, from soft, black, conducting graphitic films to hard, transparent; insulating diamondlike films [40]. Initial models of amorphous carbon were based on the assumption that graphitic films contain a high proportion of $\mathrm{sp}^{2}$ carbon-carbon molecular bonding while the diamondlike films contain a high proportion of $\mathrm{sp}^{3}$ bonding. There is general agreement that the soft, black films contain predominantly graphitic $\mathrm{sp}^{2}$ bonding, but sharp disagreement exists regarding the nature of carbon-carbon bonding in hard, transparent films [40]. The SMD of these different bonds are quite distinct and can be easily distinguished, in principle, by $(e, 2 e)$ spectroscopy. The $(e, 2 e)$ data indicates that even in graphitic amorphous carbon the nature of the carbon-carbon bonding is more subtle than present models would suggest. 
Three groups have studied graphitic amorphous carbon $[3,18,35,38]$ and one group has studied diamondlike amorphous carbon by (e,2e) spectroscopy [16]. These studies find that in both forms of amorphous carbon significant spectral weight exists near the binding energies of the $\pi$ and $\sigma$ bands of graphite. Within experimental error, the SMD of the lowest $\sigma$ band of graphite is identical to a feature at the corresponding energies in the SMD of graphitic and diamondlike amorphous carbon. But the $\pi$ band SMD in graphite is qualitatively different from the corresponding feature in graphitic and diamondlike amorphous carbon. There are electron levels in amorphous carbon with binding energies which overlap the $\pi$ bands of graphite, but the momentum densities for the amorphous carbon levels and the graphite $\pi$ band levels are qualitatively different. Thus, evidence from (e,2e) spectroscopy does not support a model which describes amorphous carbon as simply a variable mixture of graphitic $\mathrm{sp}^{2}$ and diamond $\mathrm{sp}^{3}$ bonding. We will review the experimental results for ion sputtered and evaporated amorphous carbon and then discuss the interpretations of the measurements.

\subsubsection{Evaporated amorphous carbon}

In all these studies of evaporated amorphous carbon $[3,18,35,38]$, the films were $\sim 100 \AA$ thick and were prepared by striking an arc between two high-purity graphite rods and allowing the carbon plasma to condense at room temperature on a glass slide which had been coated with a water soluble detergent [73]. The films separate from the slide when it is emersed in water and then can be mounted on sample holders.

A serious concern in studying such thin films is whether the local bonding is oriented with respect to the substrate. Grill et al. [74] found that the nature of carbon-carbon bonding in plasma deposited, hydrogenated amorphous carbon films depended upon the depth into the film from the film-substrate interface. The bonding was inhomogeneous to a depth of $400 \AA$ from the interface. If evaporated amorphous carbon tends to form $\mathrm{sp}^{2}$ bonded sheets, there might be significant alignment of these sheets with the substrate in very thin films. Since the SMD of graphite is quite 
anisotropic for momentum vectors parallel and perpendicular to the c-axis, the SMD of evaporated carbon is also likely to be anisotropic if the $\mathrm{sp}^{2}$ bonded sheets tend to align with the substrate. Kheifets et al. [38] measured the SMD of their sample with momentum vector (magnitude $1 \AA^{-1}$ ) parallel and perpendicular to the film and saw no anisotropy within their error bars. Electron diffraction studies of similarly prepared films revealed diffuse rings consistent with an amorphous structure [75]. The Raman spectra of equivalent samples also was broad and featureless [15].

A representative spectrum from Kheifets et al. of (e,2e) count rate as a function of binding energy (with respect to the Fermi energy) for $q=0$ is shown in Fig. 3(a). The solid line is the data corrected for multiple scattering events. The two peaks centered at $8 \mathrm{eV}$ and $21 \mathrm{eV}$ in this spectra are at the same binding energies as the centroid of $\pi$ and $\sigma$ band doublets at the zone center of graphite. These two features are present in all published measurements on evaporated amorphous carbon. The full data set of Kheifets et al. showing the SMD as a function of binding energy for different momenta is shown in Fig. 8(a) [38]. The energy resolution is $1.5 \mathrm{eV}$ FWHM and the momentum resolution is $0.3 \AA^{-1}$ FWHM. The measurements of Hayes et al. are shown in Fig. 8(c), where the energy and momentum resolutions are $4.5 \mathrm{eV} F W H M$ and $1.0 \AA^{-1} \mathrm{FWHM}$, respectively [35]. The measurements of Ritter et al. are shown in Fig. 8(d), where the energy and momentum resolutions are $6.0 \mathrm{eV}$ FWHM and $0.5 \AA$ A-1 FWHM, respectively [3]. The three measurements are in good agreement within their respective energy and momentum resolution. We now consider the data in more detail.

A strong feature in the spectra shown in Fig. 8 disperses from $22 \mathrm{eV}$ at $\mathrm{q}=0$ to $11 \cdot \mathrm{eV}$ at $q=1.9 \AA^{-1}$ where it coalesces with a second feature. The intensity of the first feature displays $S$ wave angular momentum symmetry decreasing monotonically with increasing momentum. The second feature is weaker than the first and is dispersionless within the experimental uncertainty.. The binding energy of this feature is approximately $10 \mathrm{eV}$ and its intensity peaks between $q=1.9 \AA^{-1}$ and $2.6 \AA^{-1}$. But the intensity does not appear to go to zero at $q=0$ as would the momentum density of a level with pure P-wave angular momentum symmetry. The question of whether or not the SMD of the second feature goes to zero at zero momentum has significant 
implications for the interpretation of the amorphous carbon spectra and will be discussed further below. The measurements of Kheifets et al. reveal a third feature, a weak shoulder at a binding energy of $5 \mathrm{eV}$ visible in spectra with $\mathrm{q} \geq 1.13 \AA^{-1}$. All three of these features were analyzed by Gaussian curve fitting to the spectra in Fig. 8(a). The energy dispersion of the features as a function of momentum is shown in Fig. 9(a). The intensity of the features as a function of momentum is shown in Fig. 9(b). The solid and dashed lines in these figures are derived from a heuristic model for the SMD of evaporated amorphous carbon which will be discussed after presenting the experimental results for ion sputtered amorphous carbon.

\subsubsection{Ion sputtered amorphous carbon}

Gao et al. studied an ion sputtered diamondlike amorphous carbon film prepared by dc magnetron sputtering (argon pressure of $36 \mathrm{mTorr}$ and RF sputtering power of $200 \mathrm{~W}$ ) [16]. The film was hard and transparent with a DC conductivity of $\leq 10^{-2} / \Omega \mathrm{cm}$ which is four or five orders of magnitude smaller than the DC conductivity of evaporated amorphous carbon.

The (e,2e) spectra as a function of binding energy for various momenta are shown in Fig. 10. The spectra were fit by two Lorentzian functions shown as solid lines in the figure. The widths of the Lorentzian peaks were independent of momentum and approximately equal to the energy resolution. The high binding energy peak disperses from $20 \mathrm{eV}$ to $15 \mathrm{eV}$ where it coalesces with the low binding energy peak. The low binding energy peak at $8 \mathrm{eV}$ does not disperse. While the two peaks are resolved, the intensity of the high binding energy peak falls off monotonically with increasing momentum and the intensity of the low binding energy peak is nearly constant. The SMD of ion sputtered amorphous carbon is qualitatively the same as the SMD of evaporated amorphous carbon.

\subsubsection{Models of evaporated and ion sputtered amorphous carbon}


The SMD of ion sputtered amorphous carbon [Fig. 10] is remarkably similar, within the experimental scatter, to the SMD of evaporated amorphous carbon (Fig. 8). The diamondlike, ion sputtered system is expected to have a much lower concentration of $\mathrm{sp}^{2}$ bonding than the graphitic, evaporated system and, yet, at first glance the degree of $\mathrm{sp}^{2}$ bonding appears to be the same in the two systems. That is, the relative intensity of the nondispersing feature at $8 \mathrm{eV}$ (associated with $\pi$ electron levels from the $\mathrm{sp}^{2}$ bonds) to the dispersing feature (which presumably has contributions from both $\mathrm{sp}^{2}$ and $\mathrm{sp}^{3}$ bonds) is qualitatively the same in ion sputtered and evaporated amorphous carbon. A straightforward prediction of the variable $\mathrm{sp}^{2} / \mathrm{sp}^{3}$ local bonding model is that the relative density of $\pi$ electron levels to $\sigma$ electron levels should be much larger in evaporated amorphous carbon than in ion sputtered carbon.

In order to quantify the relative intensities of the two features in ion sputtered and evaporated amorphous carbon, Gao et al. calculated the partial intensity $n^{\prime} \pi(q)$ and $n^{\prime} \sigma(q)$ of each feature by integrating over energy the two Lorentzian functions in Figs. 10(b) and 8(d) [16] and adding the respective intensities (weighted by $\mathrm{q}^{2}$ ) for the different momentum $0 \leq \mathrm{q}^{\prime} \leq \mathrm{q}$. In the limit $\mathrm{q} \rightarrow \infty$, the ratio $n^{\prime} \pi(q) / n^{\prime} \sigma(q)$ is $1 / 3$ for pure $s^{2}$ bonding and is zero for pure $\mathrm{sp}^{3}$ bonding. For a mixture of bonding with concentration $\mathrm{c}_{\mathrm{sp}^{2}}$ of $\mathrm{sp}^{2}$ bonding and $1-\mathrm{c}_{\mathrm{sp}^{2}}$ of $\mathrm{sp}^{3}$ bonding, the ratio is $\mathrm{n}^{\prime} \pi(\mathrm{q} \rightarrow \infty) / \mathrm{n}^{\prime}(\mathrm{q} \rightarrow \infty)=\mathrm{c}_{\mathrm{sp}^{2}} /\left(4-\mathrm{c}_{\mathrm{sp}^{2}}\right)$. Unfortunately, the $\pi$ and $\sigma$ features in the experimental data cannot be resolved for $q \geq 1.5 \AA^{-1}$, and, therefore, the $q \rightarrow \infty$ limit of the ratio cannot be determined. A model dependent analysis is necessary to determine $c_{\mathrm{sp}}$.

The partial intensities $n^{\prime} \pi$,Theor $(q)$ and $n^{\prime} \sigma$,Theor $(q)$ of the $\pi$ band in graphite and the $\sigma$ bands in graphite and diamond were calculated from an angular average of the respective theoretical SMD. The solid lines in Fig. 11 are the ratios $n^{\prime} \pi$,Theor $(\mathrm{q}) / n^{\prime} \sigma$,Theor $(\mathrm{q})$ as a function of momentum: for different relative concentrations of graphitic bonding. Notice the break in the abscissa and the exact asymptotic results for the limit $q \rightarrow \infty$. The open and solid circles are data for evaporated and ion sputtered amorphous carbon, respectively. The experimental ratio is very sensitive to statistical fluctuations in the data at low momenta, but the ratios for evaporated and ion sputtered carbon are clearly approaching the same limit, within the experimental uncertainties, as the momentum 
exceeds $q=1.5 \AA^{-1}$. The experimental ratios for both diamondlike and graphitic amorphous carbon are consistent with $100 \% \mathrm{sp}^{2}$ bonding, but given the experimental scatter, it is not possible to rule out $10 \%$ to $20 \% \mathrm{sp}^{3}$ bonding in these materials.

Given that both evaporated and ion sputtered amorphous carbon have predominantly $\mathrm{sp}^{2}$ bonding, it is reasonable to ask how well the SMD of these materials might be modeled by an angular average of the SMD of graphite. Kheifet et al. [38] suggested such a heuristic model for evaporated amorphous carbon. They calculated the SMD of graphite based on an $a b$ initio, selfconsistent linear muffin-tin orbital method. The results, which were in agreement with earlier calculations of energy bands and momentum densities in graphite [15], were then averaged over solid angle. This average, convoluted with the experimental resolution function, is compared to the experimental measurements in Fig 8. The scale factor between the experimental [Fig. 8(a)] and theoretical [Fig. 8(b)] results was determined by matching the intensities of the $21 \mathrm{eV}$ peaks in the $\mathrm{q}=0$ spectra. The major dispersing peak in the theoretical spectra is associated with the $\sigma$ bands. The asymmetry in the shape of this peak is due to the asymmetry in the dispersion of the $\sigma$ bands for momentum parallel and perpendicular to the c-axis in graphite. Excellent agreement is evident between this $\sigma$ band peak in the model SMD and the strongest feature in the experimental data.

The contribution of the $\pi$ band SMD to the model spectra is the weak bump in Fig. 8(b) which appears at $q=1.2 \AA^{-1}$ and goes through a maximum at $q=2.1 \AA^{-1}$. The intensity of the theoretical $\pi$ band SMD is significantly weaker than the theoretical $\sigma$ band SMD because of the anisotropic energy band dispersion combined with angular averaging of the dumbbell-shaped momentum density. The $\pi$ orbital momentum density is maximum in the polar regions of angular phase space where the energy density of states is low and is small over the equatorial regions of angular phase space where the energy density of states is large. The momentum density of an S-wave symmetry $\sigma$ orbital, on the other hand, has a much weaker angular dependence and so, regions of angular phase space with a high energy density of states are weighted equally with those regions having a low density of states. 
The second feature at $\mathrm{E}_{\mathrm{B}} \approx 10 \mathrm{eV}$ in the experimental spectra is clearly not modeled well by an angular average of the $\pi$ band SMD in graphite. The most obvious difference is the relatively strong spectral weight of this feature at low momentum where the model calculation has no weight. It could be argued that the finite weight at zero momentum in the experimental data is due to the finite momentum resolution. If this were true, one would expect the ratio of the intensity of the second feature to the intensity of the first feature at $q=0$ would scale with the momentum resolution. Instead, the relative intensity of the two features at $q=0$ is about the same in Figs. $8(\mathrm{a}), 8(\mathrm{c})$, and 8 (d) even though the momentum resolution of Kheifets et al. [38] is a factor of two or three better than the resolution in the measurements of Hayes et al. [35] and Ritter et al. [3].

The weak, third feature in the spectra of Kheifets et al. does qualitatively behave like the angular average of the $\pi$ band SMD of graphite (see Fig. 9), but the experimental uncertainty in the position and intensity of this feature is too large for this agreement with the model to be significant.

The level of disagreement between the SMD of amorphous carbon and an angular average of the SMD of graphite should not be surprising; that there is any agreement is noteworthy. The atomic structure of amorphous carbon, measured by diffraction experiments, is certainly not an angular average of some linear combination of graphite and diamond structures. The SMD of amorphous carbon also will not be an angular average of the crystalline SMD and one would prefer to compare experiment with first-principles calculations of the SMD, such as have been made for tetrahedrally bonded amorphous solids. But those studies were based on highly simplified models and do not readily apply to amorphous carbon with its variety of local carbon-carbon bonding. In the absence of first-principles calculations, one seeks through heuristic models, such as angular averaging the crystalline SMD, for guidance in developing better models for the electronic structure of these materials.

In summary, several lines of evidence from $(e, 2 e)$ spectroscopy on graphitic and diamondlike amorphous carbon are in strong conflict with the intuitively appealing model that amorphous carbon is a variable mixture of $\mathrm{sp}^{2}$ and $\mathrm{sp}^{3}$ bonding. The basic problem is that the SMD of graphitic and diamondlike amorphous carbon which should have very different ratios of $\mathrm{sp}^{2} / \mathrm{sp}^{3}$ 
bonds look very similar within the experimental scatter. As Kheifets et al. demonstrated, the dispersing feature in the spectra is very similar to an angular average of the sigma bands in graphite. Unfortunately, this agreement does not rule out the possibility that this feature is associated with $\mathrm{sp}^{3}$ bonding. Though no one has done the calculation, it is clear from inspecting the band structure of diamond that the dispersing feature in the $(e, 2 e)$ spectra of amorphous carbon might also be described by an angular average of the diamond SMD, if the chemical potential fell right at the top of the diamond valence band (The valence bandwidth of diamond is $\sim 21 \mathrm{eV}$ ). This leaves the second, nondispersing feature at $\sim 8 \mathrm{eV}$ as our only handle for discriminating between $\mathrm{sp}^{2}$ and $\mathrm{sp}^{3}$ bonding.

The energy of the nondispersing feature in the SMD of amorphous carbon falls right at the zone center energy of the graphite $\pi$ band. The integrated spectral weight of this feature, normalized to the spectral weight of the dispersing feature, is the same in graphitic and diamondlike amorphous and is consistent with $100 \% \mathrm{sp}^{2}$ bonding. Thus, there appears to be no difference in the relative concentration of $\mathrm{sp}^{2}$ and $\mathrm{sp}^{3}$ bonding between the two forms of amorphous carbon in contradiction to the variable bond model, as it is interpreted most-literally. But the disagreement between the variable bond model and experiment goes even deeper. Not only is the relative concentration of $\mathrm{sp}^{2}$ and $\mathrm{sp}^{3}$ bonding not variable, but the SMD of the levels at $8 \mathrm{eV}$ (nominally $\pi$ electron levels) is not consistent with pure $\mathrm{sp}^{2}$ bonding. That is, the SMD of these levels does not go to zero as $\mathrm{q} \rightarrow 0$ in either evaporated or ion sputtered carbon. Gao et al. suggested that the finite value of the SMD as $q \rightarrow 0$ might be due to rehybridization of graphitic $\pi$ and $\sigma$ levels [16].

\subsection{Aluminum/aluminum oxide}

Hayes et al. studied thin aluminum/aluminum oxide films [12,35,36]. They prepared samples for their study by evaporation of aluminum on a water soluble substrate. Analysis by Auger spectroscopy revealed a high level of oxide and carbon contamination on the surface. The transmission electron diffraction pattern for the samples had sharp Bragg rings at the aluminum 
reciprocal lattice vectors and a diffuse background from the disordered aluminum oxide component. The aluminum diffraction rings were relatively uniform in intensity indicating random orientation of the crystallites. The total film thickness was $\sim 100 \AA$ while the oxide surface layer on each side of the film was estimated to be 20 - 30 A thick.

The data were taken at an incident energy of $7.5 \mathrm{keV}$ using parallel detection as described in Section 3.1 [36]. The energy resolution was $4.5 \mathrm{eV}$ FWHM and the momentum resolution was 1.1 $\AA^{-1}$ FWHM. The spectra as a function of binding energy for momenta from -3.9 to $3.9 \AA^{-1}$ (steps of $0.8 \AA^{-1}$ ) are shown in Fig. 12. The extended spectra for $q=0 \AA^{-1}$ is shown in Fig. 3(b). The peak at $13 \mathrm{eV}$ is primarily single scattering ionization events and the broad featureless spectra below $40 \mathrm{eV}$ is due to multiple scattering. The region between $13 \mathrm{eV}$ and $40 \mathrm{eV}$ (including the peak at $31 \mathrm{eV}$ ) is a mixture of single and multiple scattering features. The authors deconvoluted the multiple scattering from the spectra by a Monte Carlo procedure and the solid line in Fig. 3(b) is the estimated single scattering spectra.

No evidence of the nearly free-electron band of aluminum is seen in the spectra. The dispersion of this band has been measured by ARPES and is known to vary quadratically with momentum from a binding energy of $11 \mathrm{eV}$ (with respect to the Fermi energy) at the Brillouin zone center to a binding energy of $1.5 \mathrm{eV}$ at the $\mathrm{X}$ point of the Brillouin zone where a $2 \mathrm{eV}$ gap exists between the first and second band [76]. Hayes et al. argue that the two dispersionless features at 13 $\mathrm{eV}$ and $31 \mathrm{eV}$ probably arise from the amorphous aluminum oxide surface layers. Their argument is based on XPS [77] and soft X-ray [78] measurements of the density of states.

The density of states of amorphous alumina valence bands is difficult to determine, but XPS and soft $\mathrm{x}$-ray measurements suggest that there are a high density of states derived from hybridized $\mathrm{O} 2 \mathrm{p}-\mathrm{Al} 3 \mathrm{p}$ levels extending down $10 \mathrm{eV}$ from the top of the valence band. This band of levels consists of two poorly resolved peaks which are roughly $3 \mathrm{eV}$ apart. Then there is a gap between these "upper valence band levels" and a narrow peak of "lower valence band levels" derived from $\mathrm{O} 2 \mathrm{~s}$ levels and centered $21 \mathrm{eV}$ below the top of the valence band. The total band width from the top of the valence band to the bottom of the 02 sand is estimated to be $\leq 27 \mathrm{eV}$ : 
If the feature at $13 \mathrm{eV}$ in the $(\mathrm{e}, 2 \mathrm{e})$ spectra is identified with the upper valence band seen in XPS and XES spectra, then the chemical potential in aluminum oxide must be just below the bottom of the conduction band (band gap is $\sim 6 \mathrm{eV}$ ). It is not unreasonable for defects to pin the chemical potential a substantial distance from the center of the gap. The separation between the upper and lower valance bands $(16 \mathrm{eV})$ agrees reasonably well with the separation between the two features in the $(e, 2 e)$ spectra and the atomic-like levels are not expected to disperse significantly with momentum. Thus, there is reasonable evidence supporting the identification of these two nondispersing features with the upper and lower valence bands in amorphous aluminum oxide. It is also reasonable that the $(e, 2 e)$ spectra is dominated by contributions from the oxide surface layers since the density of states of the nearly free electron band in aluminum is roughly five to ten times smaller than the peaks in the density of states of aluminum oxide.

The contribution of multiple scattering to the spectra in the neighborhood of the $31 \mathrm{eV}$ feature introduces significant uncertainty into analysis of the shape and intensity of this feature. In particular, the $31 \mathrm{eV}$ peak falls uncomfortably close to a double scattering event consisting of the $13 \mathrm{eV}$ ionization feature in aluminum oxide combined with the creation of a $15 \mathrm{eV}$ plasmon in aluminum. Though Hayes et al. attempted to correct for the multiple scattering contributions, the single scattering spectra will be quite uncertain below the $13 \mathrm{eV}$ peak and extensive theoretical analysis in this range of binding energy is not warranted until better data is available.

\section{THE NEXT GENERATION OF $(e, 2 e)$ SPECTROMETERS}

The two major technical problems hampering the full application of transmission (e,2e) spectroscopy to solids are low count rate and the necessarily high kinetic energies of the incident, scattered, and recoiling electrons to minimize multiple scattering. The two problems are inversely correlated since the coincidence rate is proportional to $E_{0}-7 / 2$ in the symmetric scattering geometry. One approach, which is already being utilized, is to measure the SMD at several energy-momentum points in parallel rather than in serial order. Another approach to increase the data rate is to use an 
asymmetric scattering geometry in which one detector is moved to the forward direction and captures a fast electron while the second detector is moved to a larger scattering angle and captures a slow electron $[24,25]$. The count rate is proportional to $\sin (\theta)^{-4}$ so that moving one detector from $45^{\circ}$ to $15^{\circ}$, for example, increases the date rate by over a factor of 50 . Combining this geometry with parallel detection, one obtains, in principle, over three orders of magnitude improvement in data rate. This scattering geometry looks promising, but initial experiments [24] indicate that the problem of multiple scattering is exacerbated by the short mean free path of the slow electron.

Finally, we consider an idealized spectrometer based on the symmetric scattering geometry (to minimize multiple scattering) consisting of an array of detectors at different azimuthal angles around the incident beam direction. The idea is to utilize the degeneracy of scattering geometries corresponding to the azimuthal placement of the detectors. A version of this concept was built by Moore et al. for studying atoms and molecules in gas targets [20]. The question is: how many pairs of detectors (for the scattered and ejected electrons) are required in the azimuthal ring to take a complete set of data on a sample in a few days.

First, we assume that the incident energy of an ideal spectrometer would be $-100 \mathrm{keV}$ in order to study films which are $\sim 500 \AA$ thick (the approximate mean free path for a $100 \mathrm{keV}$ electron in most materials) without being overwhelmed by multiple scattering. We seek an energy resolution of $1 \mathrm{eV}$ and a momentum resolution of $0.2 \mathrm{~A}^{-1}$. For a single energy-momentum channel, the data rate of this spectrometer is $3 \times 10^{-4}$ times the rate observed by Ritter et al., $\mathrm{N} \sim 0.02$ events/sec [3,14], using a spectrometer with an incident beam energy $\mathrm{E}_{O}=25 \mathrm{keV}$ to study a target $\mathrm{t}=100 \AA$ thick with energy and momentum resolution of $7 \mathrm{eV}$ and $0.4 \AA^{-1}$, respectively $\left(\mathrm{I} \propto \mathrm{t} \mathrm{E}_{o^{-}}{ }^{7 / 2} \Delta \mathrm{E} \Delta \mathrm{q}^{4}\right) . \quad$ Using position-sensitive detectors for both the scattered and ejected electrons and assuming each detector covers a range of energy and momentum of $10 \mathrm{eV}$ and $1 \AA^{-1}$, respectively, we obtain a factor of $10^{4}$ improvement in the data rate. The energy and momentum. range can not be too much larger because of mechanical constraints and the effects of aberrations on the electron optics. In order to obtain data on $\sim 1000$ energy-momentum points with $5 \%$. statistical accuracy requires taking data for 80 days, if one pair of such position sensitive detectors 
is used. To take the data in four days requires 20 pairs of such detectors placed around the azimuthal ring.

Is it worth the time and effort to build such a spectrometer (not necessarily the spectrometer design described above, but some approach which allows the SMD of relatively thick samples to be measured with good energy and momentum resolution in a few days)? One of the great unifying concepts in our understanding of crystalline solids is Bloch's theorem and the concomitant energy versus wavevector band dispersion. As Ashcroft and Mermin stated in an obscure footnote of their classic textbook: "Although there has been a great burst of interest in amorphous solids (starting in the late 1960's), the subject has yet to develop any unifying principles of a power even remotely comparable to that provided by the consequences of a periodic array of ions ... the subject of amorphous solids still lacks the kind of broad principles suitable for inclusion in an elementary text" [79]. The spectral momentum density, we suggest, is an ideal theoretical tool for bridging the chasm between crystalline and amorphous solids and only $(e, 2 e)$ spectroscopy can cleanly measure it.

\section{Acknowledgements}

We gratefully acknowledge major contributions to the development of (e,2e) spectroscopy at Virginia Tech by Jamie Dunn and Chao Gao. We also appreciate numerous discussions with Steve Schnatterly, E. Ward Plummer, Erich Weigold, Michael Coplan, Jack Moore, Ian McCarthy, Peter Stoner, and Anne-Marie Grisogono. Our research was supported in part by grants from the Aluminum Company of America, the Virginia Center for Innovative Technology, and by Grant No. DMR-8420555 from the National Science Foundation. 


\section{References}

1 Yu.F. Smirnov and V.G. Neudachin, Sov. Phys. - JETP Lett. 3 (1966) 192.

2 V.G. Levin, V.G. Neudachin, and Yu.F. Smirnov, Phys. Stat. Sol. (b) 49 (1972) 489.

3 A.L. Ritter, J.R. Dennison, and R. Jones, Phys. Rev. Lett. 53 (1984) 2054.

4 U. Amaldi Jr., A. Egidi, R. Marconero and G. Pizzella, Rev. Sci. Instrum. 40 (1969) 1001.

5 R. Camilloni, A.G. Guidoni, R. Tiribelli, and G. Stefani, Phys. Rev. Lett. 29 (1972) 618.

6 N.A. Krasil'nikova and N.M. Persiatseva, Soviet Tech. Phys. Lett. 1 (1975) 127.

7 J. F. Williams, S. Dey, D. Sampson and D. McBrinn, Momentum Wave Functions Conf. (Am. Inst. Phys. Topical Conf. Ser 86, 1982) pp. 314-25.

8 S. Dey and J. F. Williams, J. Phys. D: Appl. Phys. 21 (1988) 108.

9 N.M. Persiantseva, N.A. Krasil'nikova, and V.G. Neudachin, Sov. Phys. JETP 49 (1979) 530; N.A. Krasil'nikova and N.M. Persiantseva, Phys. Lett. 69A (1978) 287.

10 S. Dey, P. A. Hayes, and J. F. Williams, J. Phys. D 20 (1987) 504.

11 I.E. McCarthy and E. Weigold, Rep. Prog. Phys. 51 (1988) 299.

12 J.F. Williams and P.A. Hayes, Aust. J. Phys. 43 (1990) 465.

13 L. Frost and E. Weigold, Momentum Wave Functions Conf. (Am. Inst. Phys. Topical Conf. Ser. 86, 1982) pp. 326-331.

14 A.L. Ritter, J:R. Dennison, and J. Dunn, Rev. Sci. Instrum. 55 (1984) 1280.

15 C. Gao, A.L. Ritter, J.R. Dennison, and N.A. W. Holzworth, Phys. Rev. B 37 (1988) 3914.

16 C. Gao, Yun Yu Wang, A.L. Ritter and J.R. Dennison, Phys. Rev. Lett. 62 (1989) 945.

17 P. A. Hayes, J. F. Williams, and J. Flexman, J. Elec. Spectrosc. Rel. Phenom. 53 (1990) 5. 
18 J. Lower, S.M. Bharath, Yu Chen, K.J. Nygaard and E. Weigold, Surf. Sci. 251/252 (1991) 213; K.J. Nygaard, Y. Chen, J. Lower, P. Storer, and E. Weigold, Microscopy Microanalysis Microstructures 2 (1991) 377.

19 J. Lower and E. Weigold, J. Phys. E: Sci. Instrum., 22 (1989) 421.

20 J. H. Moore, M. A. Coplan, T. L. Skillman, Jr., and E. D. Brooks, III, Rev. Sci. Instrum. 49 (1978) 463; T. L. Skillman, E. D. Brooks, M. A. Coplan, and J. H. Moore, Nucl. Instrum. 155 (1978) 267.

21 J. P. D. Cook, I. E. McCarthy, A. T. Stelbovics and E. Weigold, J. Phys. B: At. Mol. Phys. 17 (1984) 2339.

22 I. E. McCarthy and E. Weigold Phys. Rev. A 31 (1985) 160.

23 M. A. Bennett, P. A. Smith, D. K. Waterhouse, M. J. Ford, J. Flexman, and J. F. Williams, Rev. Sci. Instrum. 63 (1992) 1922.

24 E. Weigold, J. de Physique IV 3 (1993) 187.

25 P. Storer, R. S. Caprari, S. A. C. Clark, M. Vos, and E. Weigold, Rev. Sci. Instrum 65 (1994) 2214; P. Storer, Ph.D. dissertation, Flinders University, 1993.

26 P. Hayes, M. A. Bennett, J. Flexman and J. F. Williams, Rev. Sci. Instrum. 59 (1988) 2445 .

27 P. E. Best and H. Zhu, Rev. Sci. Instrum. 56 (1985) 389.

28 B. R. Todd, N. Lermer and C. E. Brion, Rev. Sci. Instrum. 65 (1994) 349.

29 F. Bell, J. Phys. B 22 (1989) 287.

30 J. Bonfert, H. Graf and W. Nakel, J. Phys. B 24 (1991) 1423; H. Ruoff and W. Nakel, J. Phys. B 20 (1987) 2299; E. Schule and W. Nakel, J. Phys. B 15 (1982) L639; H. R. J. Walters, et al., Phys. D 23 (1992) 353.

31 A. Cavall and L. Avalei, Il Nuovo Cimento 16 (1994) 1.

32 J. Kirschner, O. M. Artamonov, and A. N. Terekhov, Phys. Rev. Lett. 69 (1992) 1711. Also refer to Ref. 27.

33 I.E. McCarthy and E. Weigold, Adv. in Phys. 25 (1976) 489. 
34 E. Weigold and I.E. McCarthy, Ad. At. and M. Phys. 14 (1978) 127.

35 P. Hayes, J.F. Williams and J. Flexman, Phys. Rev. B 43 (1991) 1928.

36 P. Hayes, M.A. Bennett, J. Flexman, and J.F. Williams, Phys. Rev. B 38 (1988) 13371.

37 See, for example; J,-C. Charlier, X. Gonze, and J.-P. Michenaud, Phys. Rev. B 43 (1991) 4579 , and references therein.

38 A. S. Kheifets, J. Lower, K. J. Nygaard, S. Utteridge, M. Vos, E. Weigold and A. L. Ritter, Phys. Rev. B 49 (1994) 2113.

39. N. A. W. Holzwarth, S. G. Louie, and Shrab Rabii, Phys. Rev. B 26 (1982) 5382.

40 J. Robertson, Adv. Phys. 35 (4) (1986) 317; J. Robertson, Prog. Solid St. Chem. 21 (1991) 199.

41 There are two major kinematic divisions based on scattering geometry, the symmetric and asymmetric $\left(\theta_{1} \neq \theta_{2}\right)$ geometries. There are two subdivisions within the symmetric geometry, coplanar $(\phi=0)$ and non-coplanar $(\phi \neq 0)$. Refer to Ref. 33 for further details.

42 The dependence of the $(\mathrm{e}, 2 \mathrm{e})$ cross section on $\mathrm{E}_{\mathrm{B}}(\mathbf{q})$ is contained in the delta function in eqn. 5. Levin et al. [2] show there is no energy dependence for non-dispersive bands while for nearly-free electrons with effective mass $m^{*}$, the correction is $\sim\left[1+\left(\mathrm{m} / \mathrm{m}^{*}\right) \mathrm{q} / \mathrm{P}_{0}\right]$ where $q \leq 4 \AA^{-1}$ in most (e,2e) experiments on solids and $P_{0}=80 \AA^{-1}$ for $E_{0}=25 \mathrm{keV}$. There is a weak dependence of the Mott cross section on q for some scattering geometries.

43 U. Amaldi, Jr. and C. C. d. Atti, Nuovo Cimento, 66A (1970) 129.

44 I.E. McCarthy and E. Weigold, Rep. Prog. Phy. 54 (1991) 789.

45 L.J. Allen, I.E. McCarthy, V.W. Maslen and C.J. Roussow, Aust. J. Phys. 43 (1990) 453.

46 V.W. Maslen and C.J. Roussow, Philos. Mag. A 49 (1984) 735.

47 C.J. Roussow and V.M. Maslen, Philos. Mag. A 49 (1984) 743.

48 I.E. McCarthy, Aust. J. Phys. 43 (1990) 419.

49 In free-electron metals, band gaps are of order $1 \mathrm{eV}$ or less and the nonparabolic dispersion is confined to a region of order $0.1 \AA^{-1}$ in reciprocal space. Therefore, this effect is not observable 
in lower resolution experiments performed to date where instrumental broadening smooths out the dip. However, the band gap and nonparabolic dispersion may be observed in higher resolution experiments in progress [24].

50 For a review of the continuous random-network model and of different models for the electronic structure of amorphous solids, see Richard Zallen, The Physics of Amorphous Solids, (Wiley, New York, 1983) and S. R. Elliot, Physics of Amorphous Materials 2nd ed., (Wiley, New York, 1990).

51 J.F. Sadoc and R. Mosseri, Phil. Mag. B 45 (1982) 467.

52 D.P. DiVincenzo, R. Mosseri, M.H. Brodsky, and J.F. Sadoc, Phys. Rev. B 29 (1984) 5934.

53 R. Haydock, in Current Trends in the Physics of Materials, ed. M. Yussouff (World Scientific, Singapore, 1987), p. 370; J. Non-Cryst. Sol. 75 (1985) 121.

54 A. Gibson and R. Haydock, J. Non-Cryst. Sol. 164 (1993) 1135; Phys. Rev. Lett. 69 (1992) 3793.

55 J.L. Beeby and T.M. Hayes, Phys. Rev. B 32 (1985) 6464.

56 B.J. Hickey and G.J. Morgan, J. Phys. C: Solid State Phys 19 (1986) 6195.

57 F. Wooten and D.L. Weaire, J. Non-Cryst. Solids 64 (1984) 325.

58 S.K. Bose, K. Winer, and O.K. Andersen, Phys. Rev. B 37 (1988) 6262.

59 These initial results were interpreted (Refs. 3,35 ) as consistant with qualitative arguments by Ziman for the existence of energy bands in amorphous solids. J.M. Ziman, J. Phys. C: Solid St. Phys. 4 (1971) 3129.

60 B. Welber and M.H. Brodsky, Phys. Rev. B 16 (1977) 3660. When Welber and Brodsky refer to "crystal band structure," they are referring to the $E_{B}$ vs. $\mathbf{k}$ band dispersion relations. It is generally accepted, both experimentally and theoretically, [50] that energy bands exist in amorphous semiconductors in the sense that there are regions where the density of states is zero separating the regions where it is finite. What is more controversial is the idea that vestiges of the crystalline $\mathrm{E}_{\mathrm{B}}(\mathrm{k})$ vs. $\mathbf{k}$ band dispersion relations exist in amorphous solids. 
61 R. Jones and A.L. Ritter, J. Elec. Spectrosc. Rel. Phenom. 40 (1986) 285; A. L. Ritter, ibid., to be published:

62 H. Boersch, Z. Physik 139 (1954) 115.

63 J.A. Simpson and C.E. Kuyatt, J. Appl. Phys. 37 (1966) 3805.

64. Jones and Ritter [61] stated that the loss spectrum below the elastic peak is proportional to the $\mathrm{S}(\mathrm{E}, \mathbf{q})$ averaged over momentum. This is not correct. The loss spectrum is proportional to a function $S^{\prime}(E)$ which is closely related to a momentum average of $S(E, q)$ and which depends implicitly on the same parameters as $S(E, q)$. The function $S^{\prime}(E)$ is discussed in Ref. 61 .

65 C. E. Kuyatt and J. A. Simpson, Rev. Sci. Instrum. 38 (1967) 103.

66 D. Roy and D. Tremblay, Rep. Prog. Phys. 53 (1990) 1621.

67 A. V. Crewe, Phil. Trans. Royal Soc. London B261 (1971) 61.

68 Peter J. Goodhew, Specimen Preparation for Transmission Electron Microscopy of Materials, (Oxford University Press, New.York, 1984); Peter J. Goodhew , Practical Methods in Electron Microscopy Vol. 11, ed. by Audrey M. Glauert, (Elsevier, New York, 1985).

69 J. Fields, Ph.D. Thesis, Princetòn, 1976. J. Fink, Adv. in Electronics and Electron Physics 75 (1989) 121.

70 J. Dieleman and F. H. M. Saunders, Solid State Technol. 27 (1984) 191.

71 C. Gao, Ph.D. dissertation, Virginia Tech, 1988.

72 A. R. Law, M. T. Johnson and H. P. Hughes, Phys. Rev. B 34 (1986) 4289 and other references sited in Ref. 15.

73 J. O. Stoner, Jr., J. Appl. Phys. 40 (1969) 707.

74 A. Grill, B. S. Meyerson, V. V. Patel, J. A. Reimer and M. A. Petrich, J. Appl. Phys. 61 (1987) 2874.

75 J.C. Pivin and A. L. Ritter, to be published.

76 Harry J. Levinson, F. Greuter; and E. W. Plummer, Phys. Rev. Rev. B27 (1983) 727.

77 A. Balzrotti and A. Biaconi, Phys. Stat. Solids (b) 76 (1976): 689.

78 I. A. Brytov and Yu N. Romashchenko, Sov. Phys. Solid State 20 (1978) 384. 
79 N. W. Ashcroft and N. D. Mermin, Solid State Physics (Holt, Rinehart and Winston, New York, 1976), p. 62. 
APPENDIX: Functional dependence of $(e, 2 e)$ count rate

We repeat the heuristic derivation of Amaldi and Atti [43] for the functional dependence of the (e,2e) count rate on momentum density cut-off $q_{c}$, incident energy $E_{0}$, and momentum and energy resolution $\Delta \mathrm{q}$ and $\Delta \mathrm{E}$, respectively. Their approach is to calculate the contribution to the singles $\left(e, e^{\prime}\right)$ scattering rate from the target orbital of interest and then find the fraction of this rate giving the $(e, 2 e)$ coincidence rate. We assume the same scattering geometry discussed in Section 2.1 and take as a model for the momentum density $|\varnothing(\mathbf{q})|^{2} \propto \mathrm{qc}_{\mathrm{c}}^{-3}$ for $\mathrm{q} \leq \mathrm{q}_{\mathrm{c}}$ and $|\varnothing(\mathbf{q})|^{2}=0$ for $\mathrm{q}>$ $\mathrm{q}_{c}$. We further assume that for the singles scattering calculation particle 1 is detected and particle 2 is ejected from the orbital of interest and is not detected. In the Born approximation, the singles scattering rate is

$$
\mathrm{W}_{\text {Singles }} \propto \sum \mid \mathrm{VH}^{2} \rho\left(\mathrm{E}_{1}, \mathrm{E}_{2}, \mathrm{E}_{\text {Recoil }}\right)
$$

where the sum is an average over initial states and a sum over final states. In the plane-waveimpulse approximation

$$
\mid \mathrm{V}^{2} \propto \frac{\left|\phi\left(\mathbf{q}=\mathbf{P}_{1}+\mathbf{P}_{2}-\mathbf{P}_{o}\right)\right|^{2}}{\left|\mathbf{P}_{1}-\mathbf{P}_{d}\right|^{4}}
$$

and assuming $\mathrm{E}_{\text {Recoil }} \sim 0$

$$
\rho\left(\mathrm{E}_{1}, \mathrm{E}_{2}\right) \propto \mathrm{P}_{1} \mathrm{~d} \mathrm{E}_{1} \mathrm{~d} \Omega_{1} \mathrm{P}_{2}^{2} \mathrm{dP}_{2} \mathrm{~d} \Omega_{2} \delta\left(\mathrm{E}_{1}+\mathrm{E}_{2}-\mathrm{E}_{\mathrm{o}}-\mathrm{E}_{\mathrm{B}}\right)
$$

where $E_{B}$ is the binding energy of the orbital being studied. Since the ejected particle 2 is not detected in the singles rate, the sum over final states includes a sum over $\mathbf{P}_{2}$. The energy delta function reduces the 3-dimensional integral to a surface integral and we find

$$
\mathrm{W} \propto \frac{\mathrm{dE}_{1} \mathrm{~d} \Omega_{1}}{\left|\mathbf{P}_{1}-\mathbf{P}_{o}\right|^{4}} \frac{\mathbf{P}_{1}}{\mathrm{P}_{2}} \int \mathrm{P}_{2}^{2} \mathrm{~d} \Omega_{2}\left|\phi\left(\mathbf{q}=\mathbf{P}_{1}+\mathbf{P}_{2}-\mathbf{P}_{o}\right)\right|^{2}
$$

where $P_{2}=\sqrt{2\left(E_{o}+E_{b}-E_{1}\right)}$ in atomic units. The scattering geometry is chosen such that $P_{1}=P_{2}$ for the orbital of interest. Given our model of the momentum density, the surface integral, then, is proportional to $1 / \mathrm{q}_{c}$. Ultimately, in the (e,2e) coincidence measurement we want to measure $\mathbf{q}$ with an uncertainty $\Delta \mathbf{q}$. In order to achieve this precision, the magnitude of the solid angle must be of order $\left(\Delta q / P_{1}\right)^{2}$. Dividing by the incidence flux $\left(\propto \mathrm{P}_{0}\right)$, the singles scattering rate from a single orbital is 


$$
\mathrm{I}_{\text {singles }} \propto \eta \mathrm{t} \frac{1}{\left|\mathbf{P}_{1}-\mathbf{P}_{o}\right|^{4}} \frac{\mathrm{q}_{c}}{\mathrm{P}_{o} \mathrm{P}_{1} \mathrm{P}_{2}} \Delta \mathrm{E}_{1}\left(\frac{\Delta \mathrm{q}}{\mathrm{q}_{c}}\right)^{2}
$$

where $\eta$ is the target density, $t$ is the target thickness, and $\Delta E_{1}$ is the energy resolution. The ejected electron 2 comes out in a cone with solid angle of order $\left(\mathrm{q}_{c} / \mathrm{P}_{2}\right)^{2}$. In order to determine $\mathbf{q}$ in the $(e, 2 e)$ measurement with the desired precision $\Delta \mathbf{q}$, a fraction $\left(\Delta q / q_{c}\right)^{2}$ of the ejected electrons are detected with a second detector of solid angle $\left(\Delta \mathrm{g} / \mathrm{P}_{2}\right)^{2}$. The count rate for $(\mathrm{e}, 2 \mathrm{e})$ scattering is then

$$
\mathrm{I}_{(\mathrm{e}, 2 \mathrm{e})} \propto \frac{\eta \mathrm{t} \mathrm{q}_{c} \Delta \mathrm{E}_{1}}{\mathrm{E}_{o}^{7 / 2}}\left(\frac{\Delta \mathrm{q}}{\mathrm{q}_{c}}\right)^{4}
$$

where in the symmetric scattering geometry, $\mathrm{P}_{o}, \mathrm{P}_{1}, \mathrm{P}_{2}$, and $\mid \mathbf{P}_{1}-\mathbf{P}_{o} l$ are all of order $\sqrt{\mathrm{E}_{o}}$. 


\section{Figure Captions}

Fig. 1 Limiting examples of the (e,2e) cross section for solids. The upper curve illustrates a nearly-free electron distribution with $\mathrm{E}_{\mathrm{B}} \approx \hbar^{2} \mathrm{q}^{2} / 2 \mathrm{~m}+\mathrm{E}(0)$ except near the Brillouin zone boundary. The lower curve illustrates a tight-binding distribution derived from nondegenerate $1 \mathrm{~s}$ atomic orbitals with nearly constant binding energy $\mathrm{E} \approx \mathrm{E}_{\mathrm{a}}$. The solid curve shows the amplitude of the SMD. The dashed curve shows the dispersion relation $\mathrm{E}_{\mathrm{B}}(\mathrm{q})$ projected onto the $\mathrm{E}_{\mathrm{B}}, \mathbf{q}$ plane. The instrumental broadening is represented by the Gaussian curves.

Fig. 2(a) Kinematics for symmetric non-coplanar (e,2e) scattering. The incident, scattered and recoiling electrons have energies $\mathrm{E}_{0}, \mathrm{E}_{1}$ and $\mathrm{E}_{2}$ and momenta $\mathbf{P}_{0}, \mathbf{P}_{1}$ and $\mathbf{P}_{2}$, respectively. The momentum vectors $\mathbf{P}_{1}$ and $\mathbf{P}_{2}$ define the scattering plane. (b) Momentum conservation diagram for non-symmetric coplanar (e,2e) experiments, showing the sampled binding momentum $\mathbf{q}$.

Fig. 3 The effects of multiple scattering events in extended (e,2e) data of the coincidence counts as a function of binding energy (with respect to the Fermi energy) for momentum $q=0$ from (a) evaporated amorphous carbon (Ref. 38) and (b) aluminum/aluminum oxide (Ref. 36). The solid lines are the "true" $(e, 2 e)$ spectra with the multiple scattering deconvolved from the data.

Fig. 4 (a) Quasi-elastic scattering at $\mathrm{E}_{0}=25 \mathrm{keV}$ and $\theta=45^{\circ}$ from evaporated amorphous carbon used to estimate the parameters in the multiple scattering function. The elastic peak is at $\mathrm{E}_{\text {loss }}=0$ and the main contribution to multiple scattering due to bulk plasmon creation is centered at $\sim 23 \mathrm{eV}$. Inset (b) shows a typical time coincidence spectrum. $\Delta \mathrm{t}_{\mathrm{c}}, \Delta \mathrm{t}_{\mathrm{b}}$ and $\mathrm{t}_{0}$ are the coincidence timing window, background timing window, and coincidence delay shift, respectively. After Ref. 38. 
Fig. 5 Two (e,2e) spectrometers for scattering from thin film solid targets (a) Spectrometer of Ritter et al. [14] with serial data collection. (b) Spectrometer of Lower, et al. [18] with parallel data collection for both scattered and recoiling electrons: (EG) electron gun, (FC) Faraday cup, (QD) quadrupole electrostatic deflector, (HSA) hemispherical analyzer, (MCP) microchannel plates, and (RA) resistive anode.

Fig. 6 (a) Brillouin zone and theoretical band structure of graphite [39]. (b-d) Spectral momentum densities for different graphite bands as a function of momentum. (b) Momentum densities of the $\sigma_{1}$ and $\pi$ bands for momentum parallel to the $c$ axis $(\Gamma-A)$. Momentum densities of the $\sigma_{2}$ and $\sigma_{3}$ bands are zero by symmetry. (c) Momentum densities of the $\sigma_{1}$ and $\sigma_{2}$ bands for momentum in the $\Gamma-K$ direction. Momentum density of the $\pi$ band is zero by symmetry. (d) Momentum densities for $\sigma_{1}, \sigma_{2}$, and $\sigma_{3}$ bands for momentum in the $\Gamma-M$ direction. Momentum density of the $\pi$ band is zero by symmetry.

Fig. 7 The spectral momentum density of graphite as a function of energy (with respect to the Fermi energy) for different momenta. Symbols show data points which have been corrected for multiple scattering. The height of the bars indicates the relative intensity of the calculated SMD. The widths of the bars are equal to the splitting of the respective band doublets. (a) Momentum perpendicular to c-axis (parallel component is $-0.61 \AA^{-1}$ ). (b) Momentum parallel to the c-axis (perpendicular component is $-0.35 \AA^{-1}$ ). After Ref. 15 .

Fig. 8 The spectral momentum density of graphitic arc-evaporated amorphous carbon as a function of energy (with respect to the Fermi energy) for different momenta. Data have been corrected for multiple scattering. (a) Measurements by Kheifets et al. $\left(\Delta \mathrm{E}_{F W H M}=1.5 \mathrm{eV}\right)[38]$. (b) Theoretical calculations of the SMD of amorphous carbon based on a spherical average of the $\mathrm{SMD}$ of crystalline graphite [38]. (c) Measurements of Hayes et al. $\left(\Delta \mathrm{E}_{\mathrm{FWHM}}=4.5 \mathrm{eV}\right)$ [35]. (d) 
Measurements of Ritter et al. $\left(\Delta \mathrm{E}_{\mathrm{FWHM}}=6.0 \mathrm{eV}\right)$ [3]. Dashed lines in (c) and (d) indicate the energy-momentum dispersion of the bands.

Fig. 9 (a) Binding energies of peaks for the three bands in the SMD of arc evaporated amorphous carbon as a function of momentum based on the data of Kheifets et al. [38] shown in Fig. 8(a). (b) Integrated peak intensities for the three bands as a function of momentum. The solid and dashed lines are based on the theoretical calculations of the SMD of amorphous carbon shown in Fig. 8(b).

Fig. 10 The spectral momentum density of diamondlike ion-sputtered amorphous carbon as a function of energy (with respect to the Fermi energy) for different momenta. Solid lines are fits of two Lorentzians with the data at four different momenta. After Ref. 16.

Fig. 11 The ratio of the partial integrated spectral intensities for the " $\pi$ " and " $\sigma$ " bands of graphitic arc evaporated amorphous carbon (open circles) and diamondlike ion-sputtered amorphous carbon (solid circles) as a function of momentum. The solid lines are theoretical predictions of this ratio for different concentrations of $\mathrm{sp}^{2}$ carbon bonding [16].

Fig. 12 The spectral momentum density of an aluminum/aluminum oxide film as a function of energy (with respect to the Fermi energy) for different momenta measured by Hayes et al. [35]. Dashed lines indicate the lack of energy-momentum dispersion. The data have been corrected for multiple scattering. 


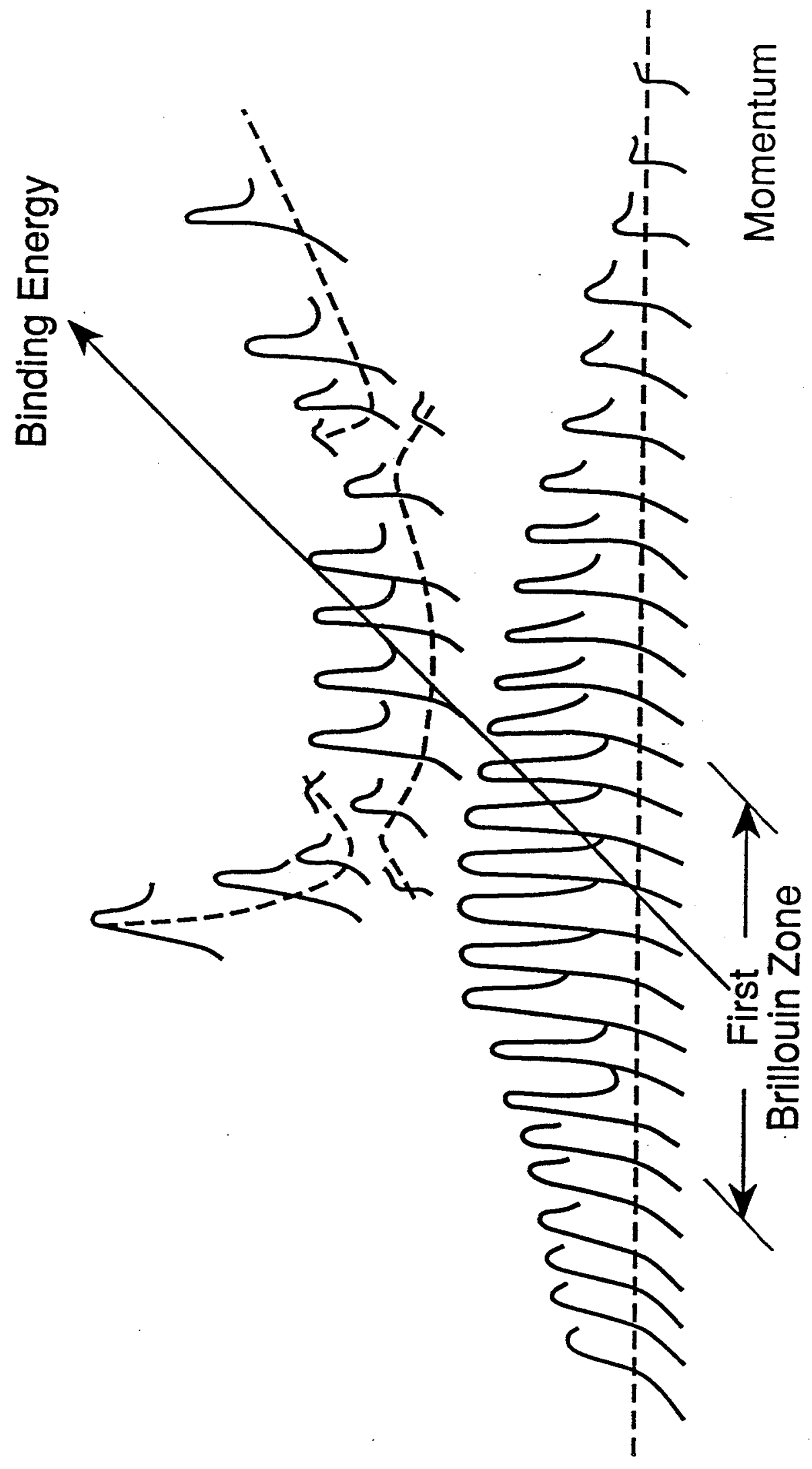




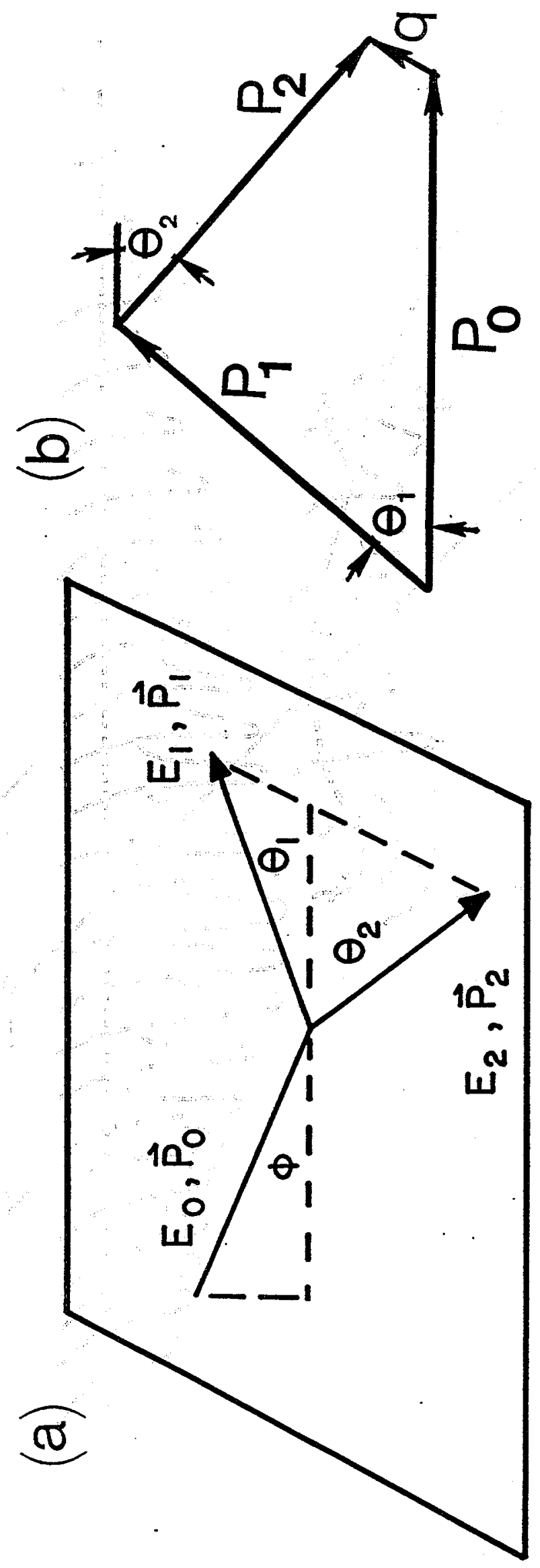

is 

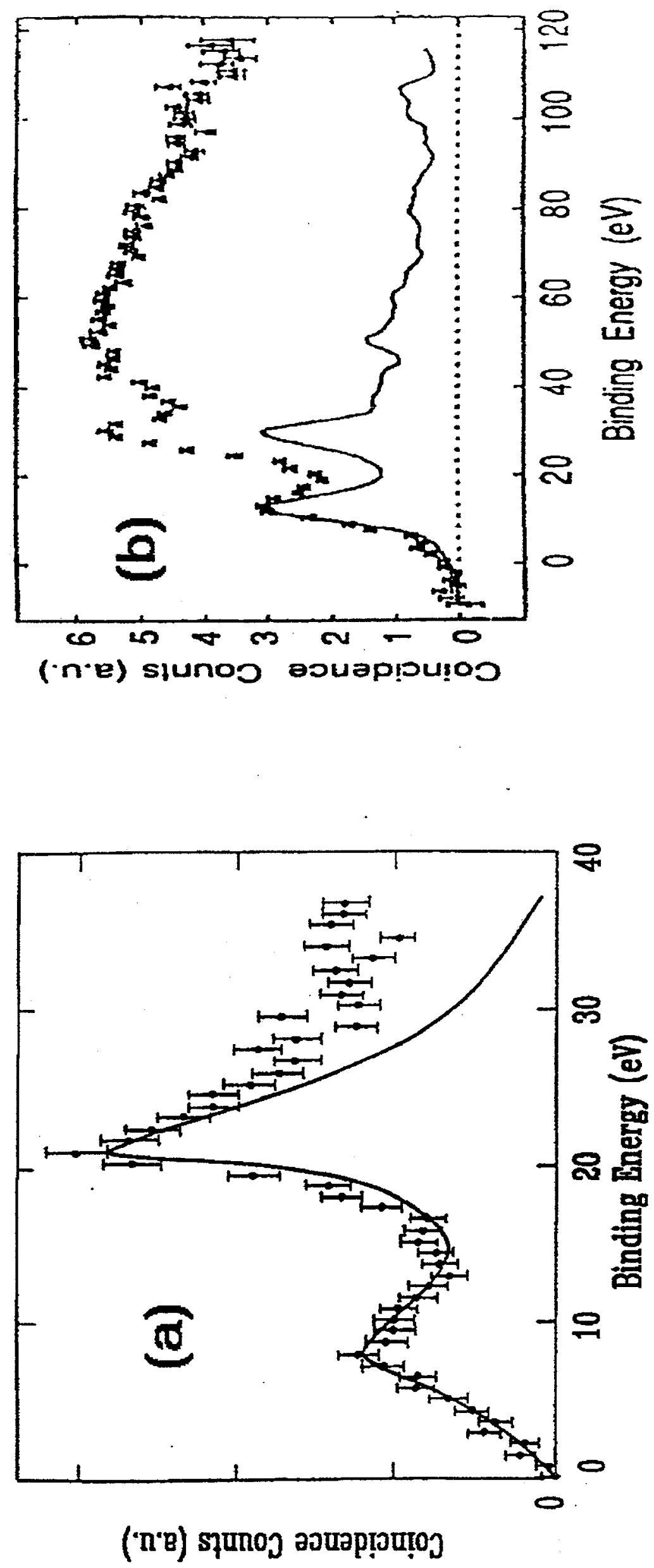


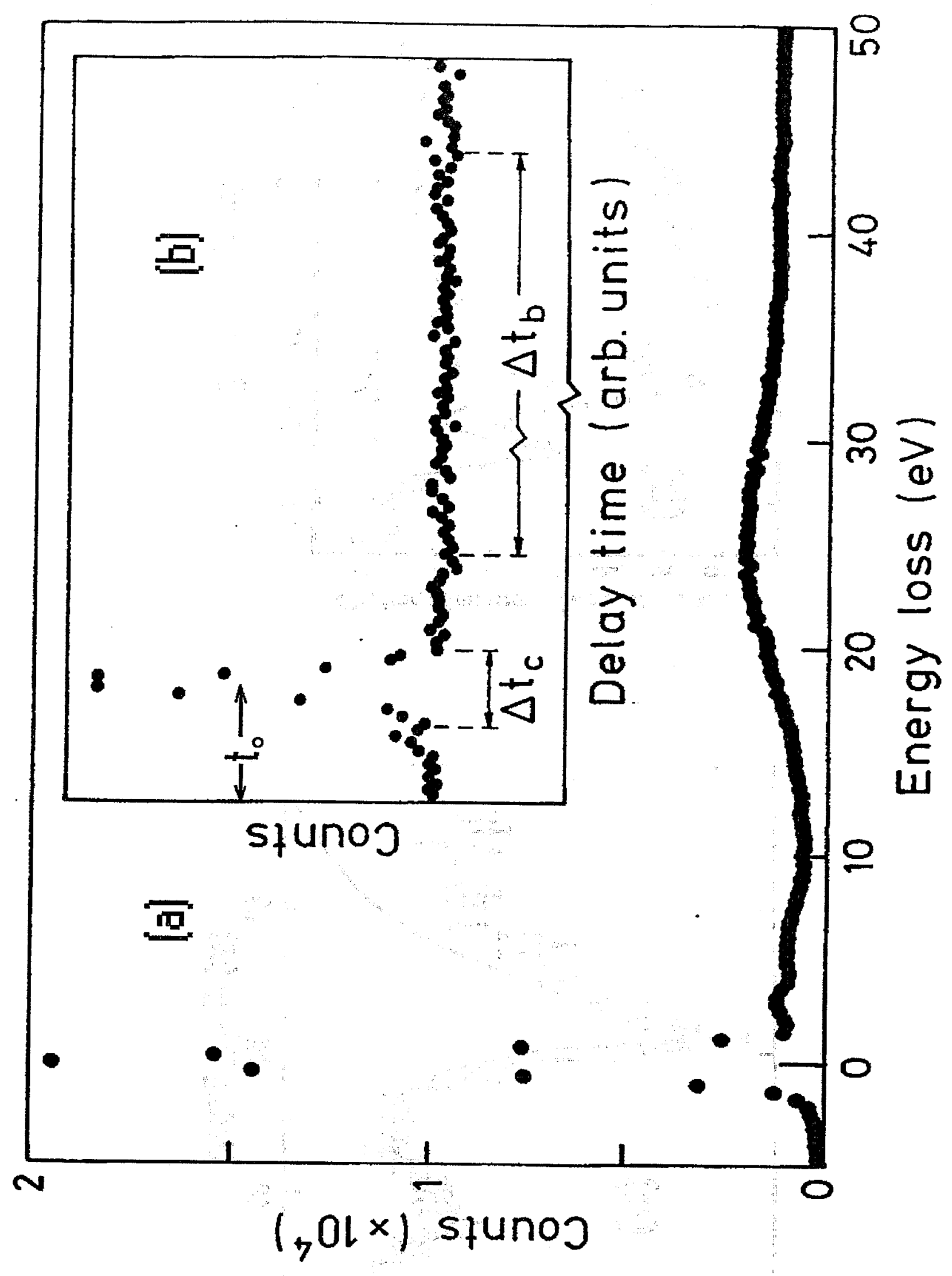



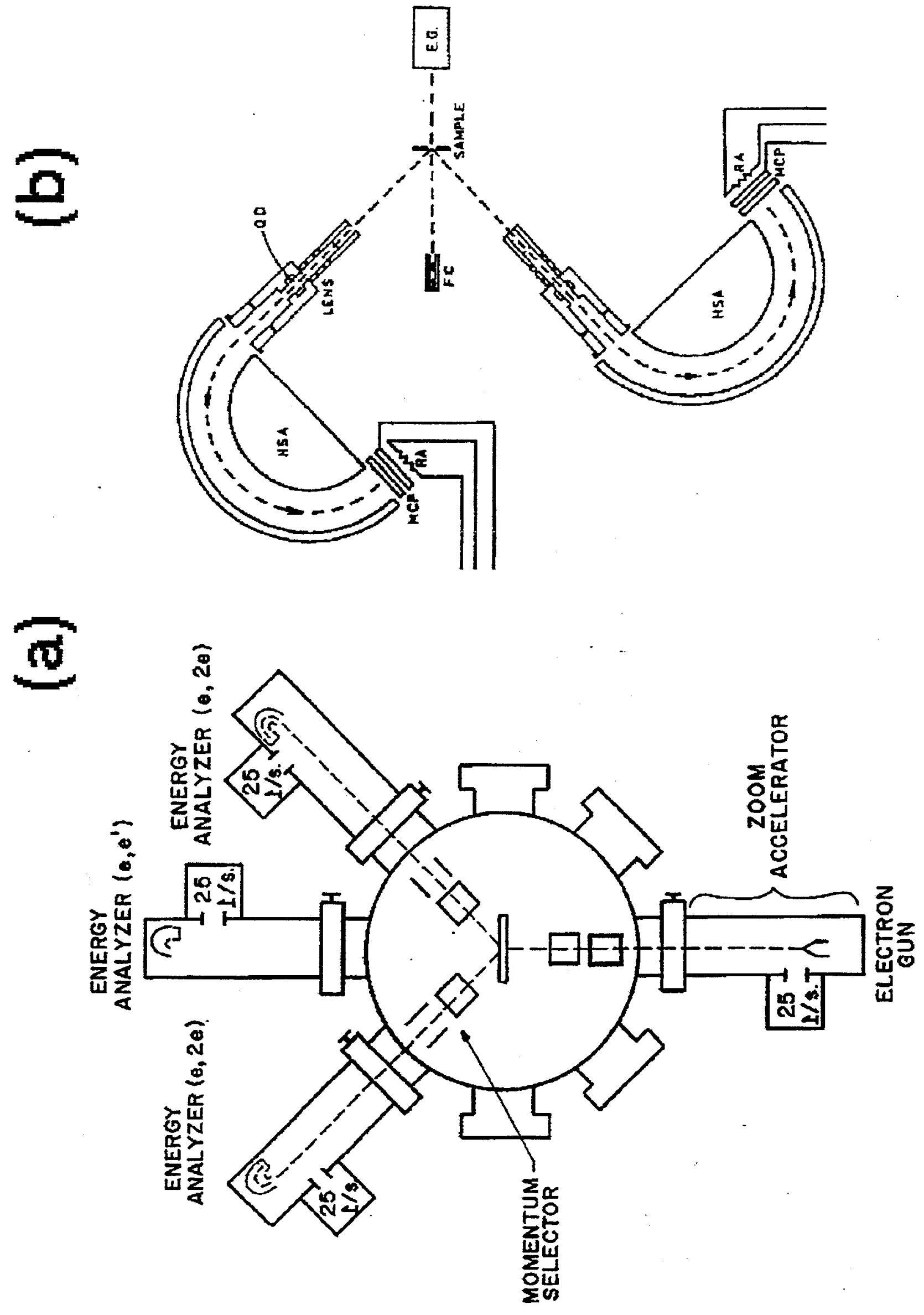

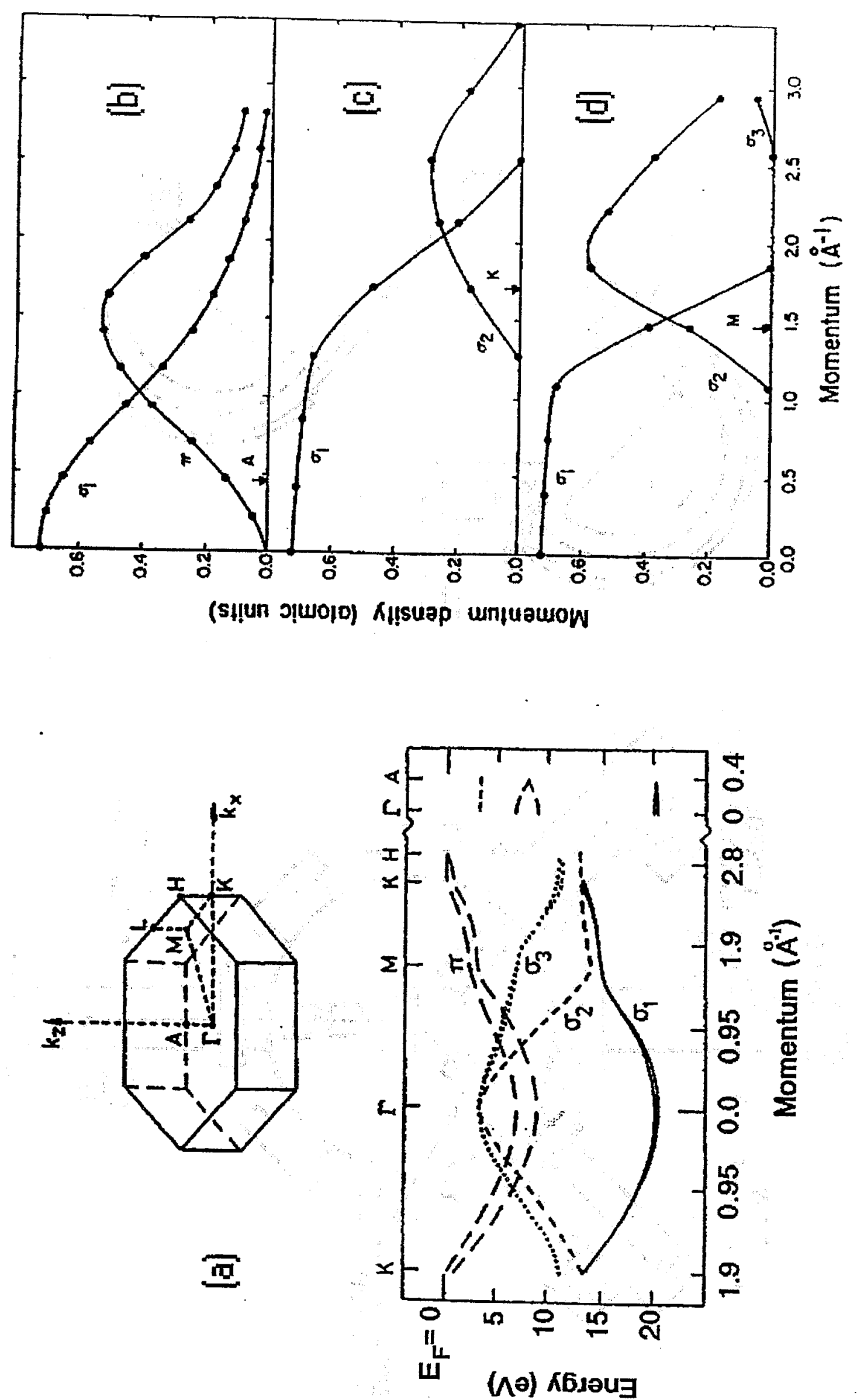

के 

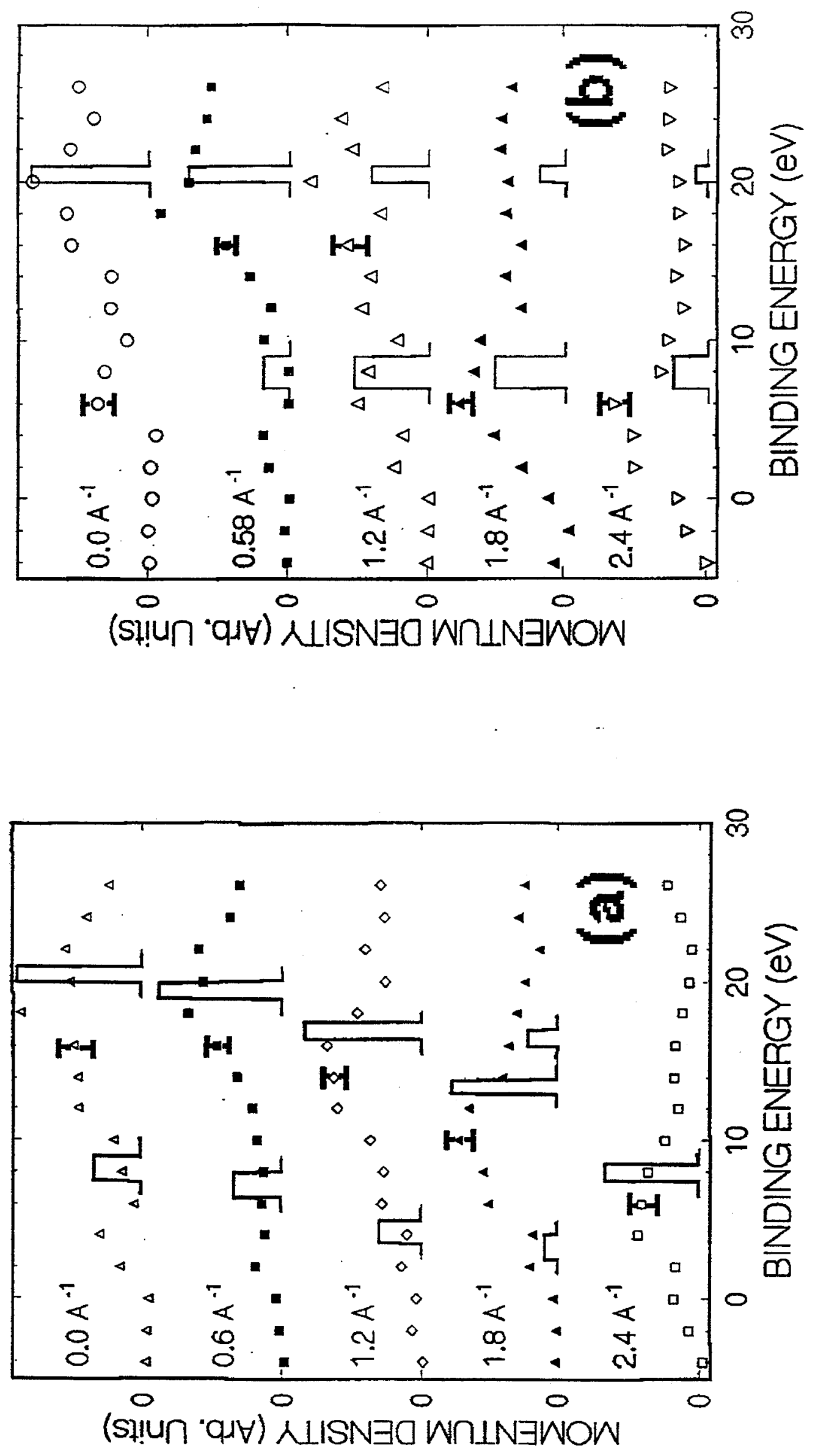

$M$ 

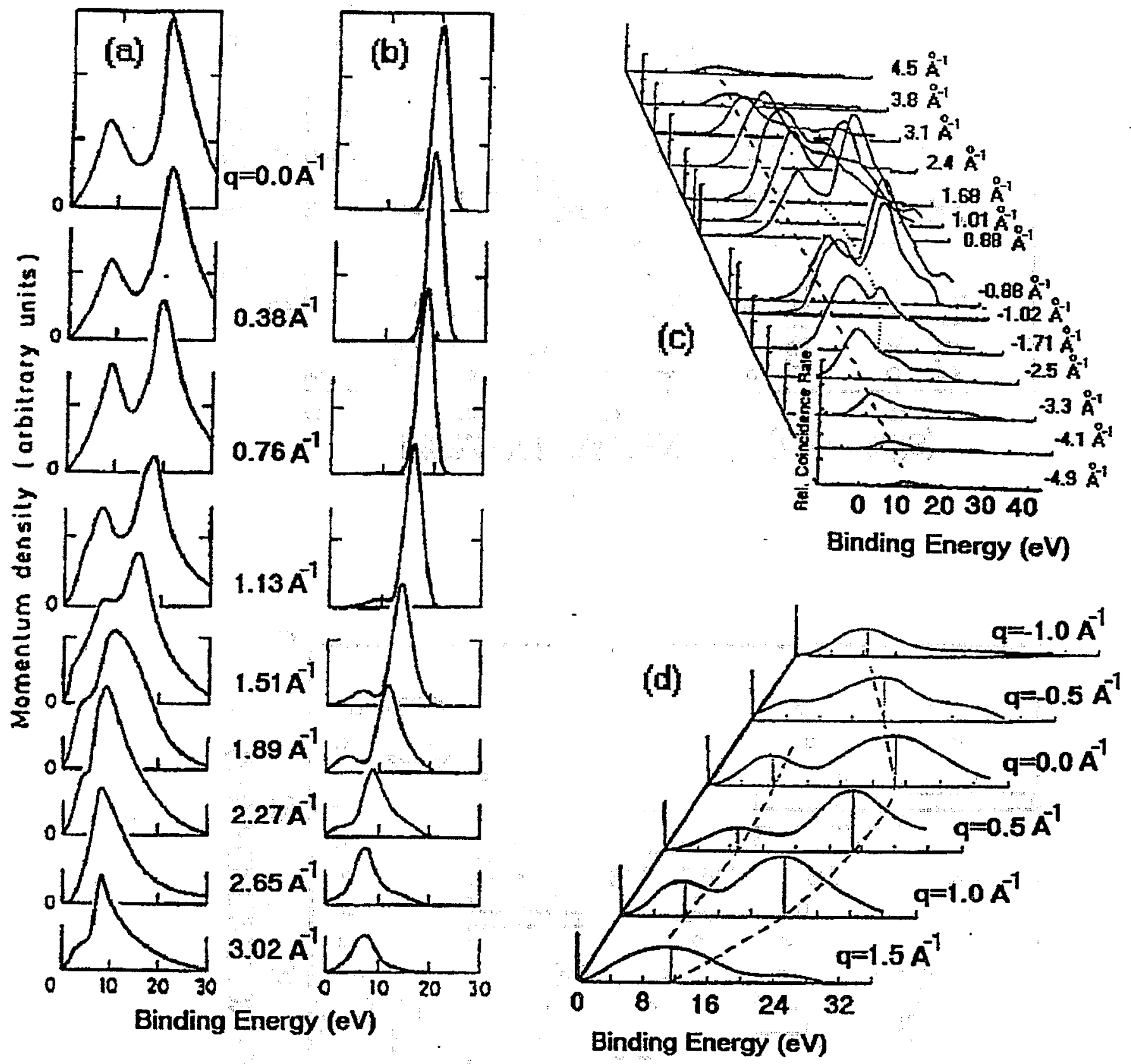

$F_{\text {In }} \&$ 

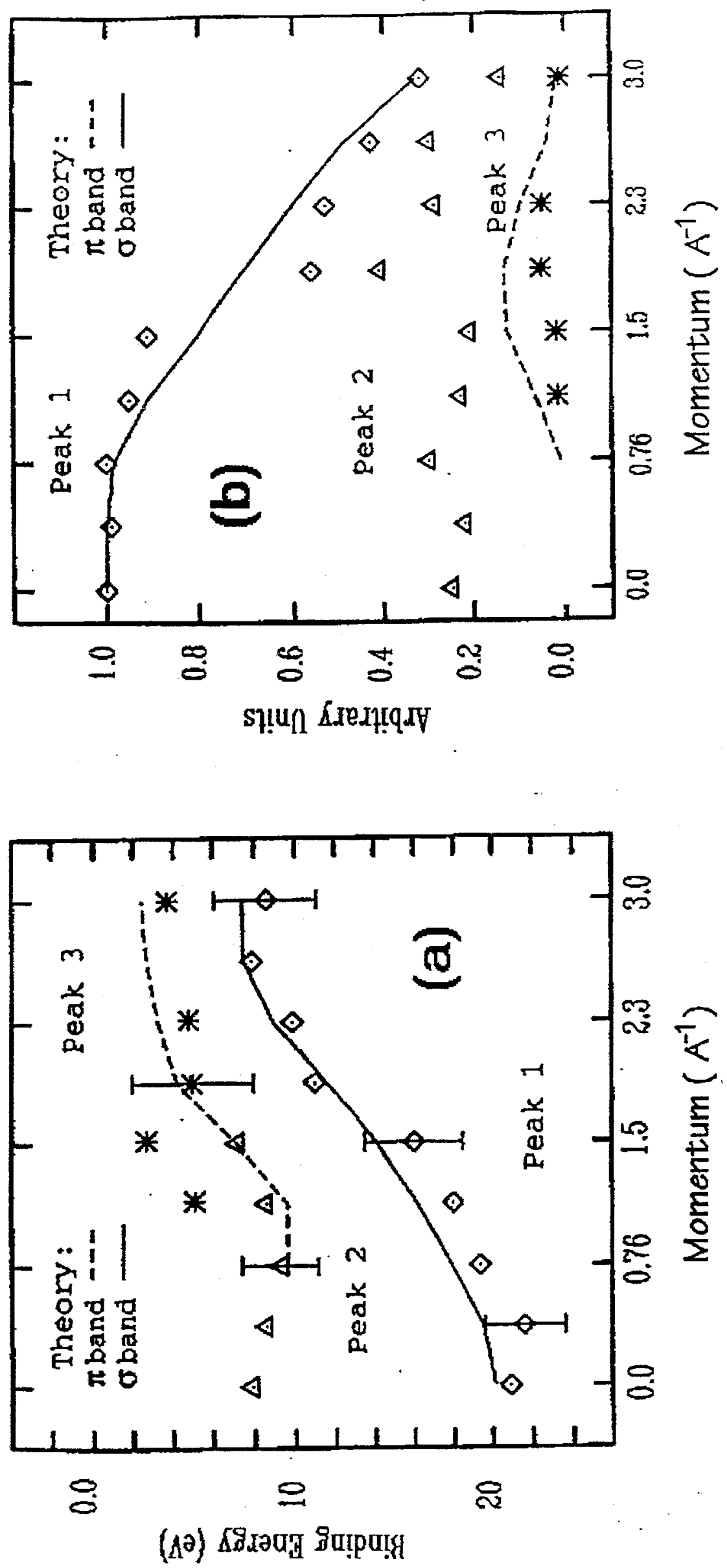


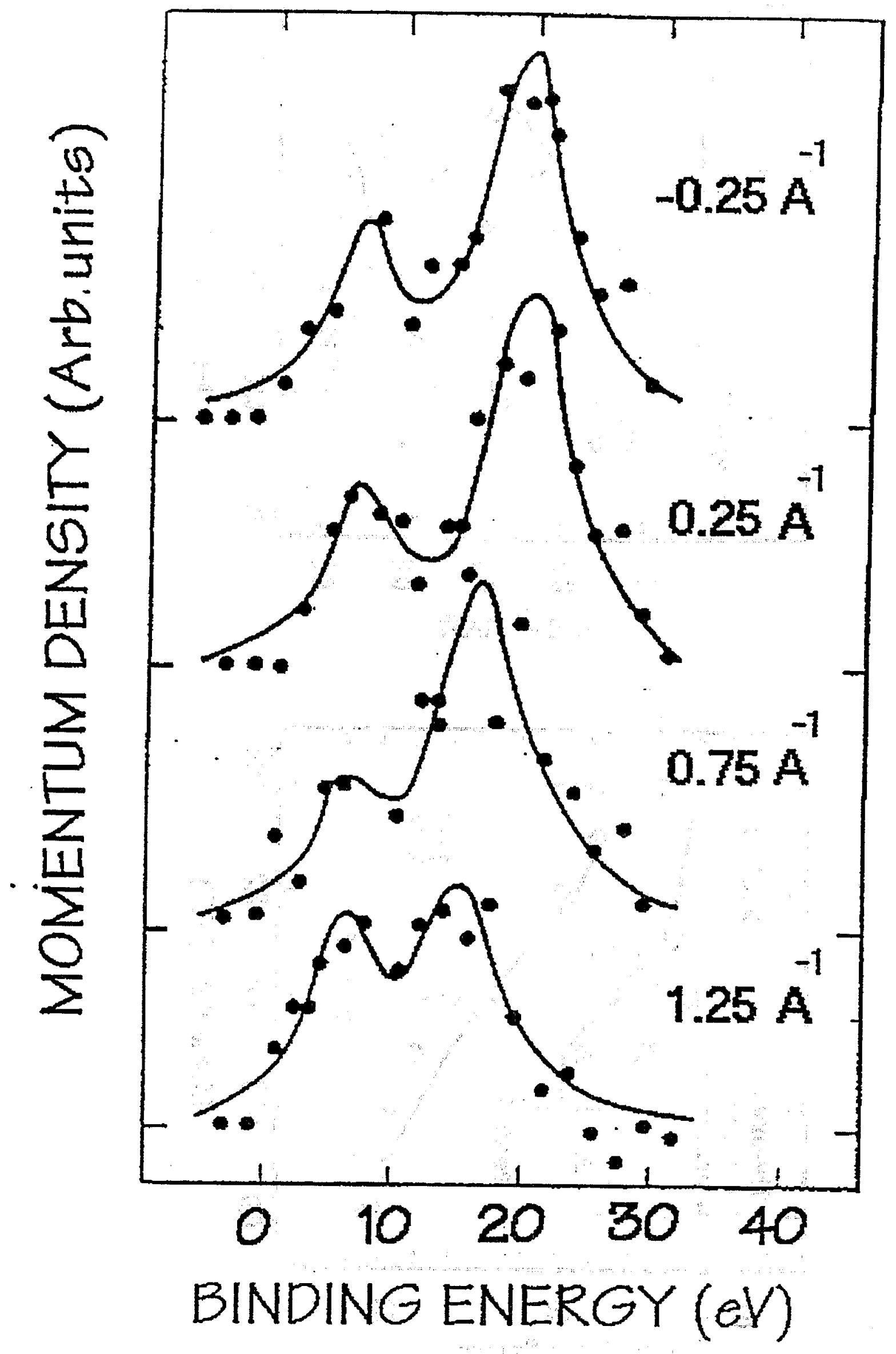

E. in 


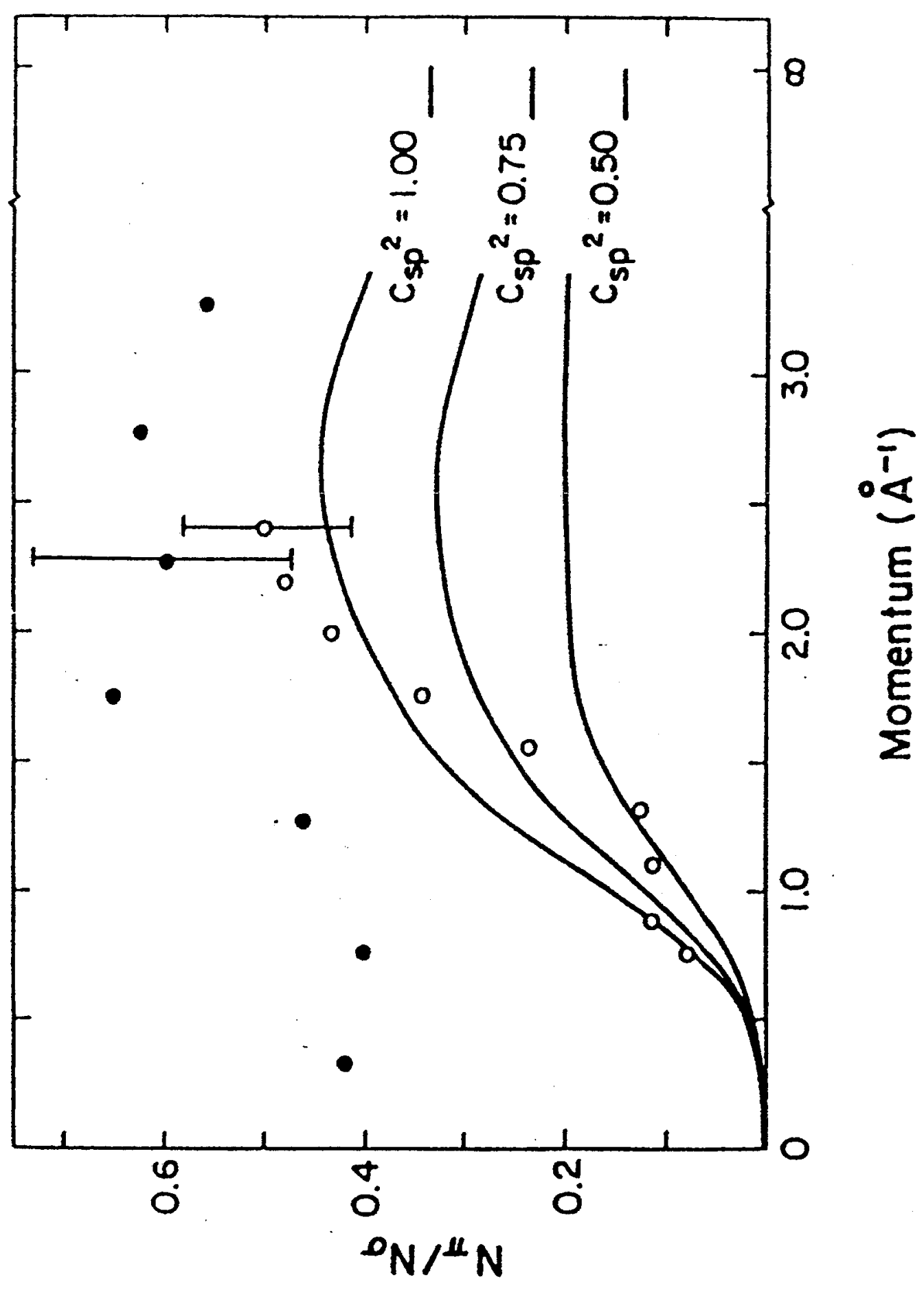




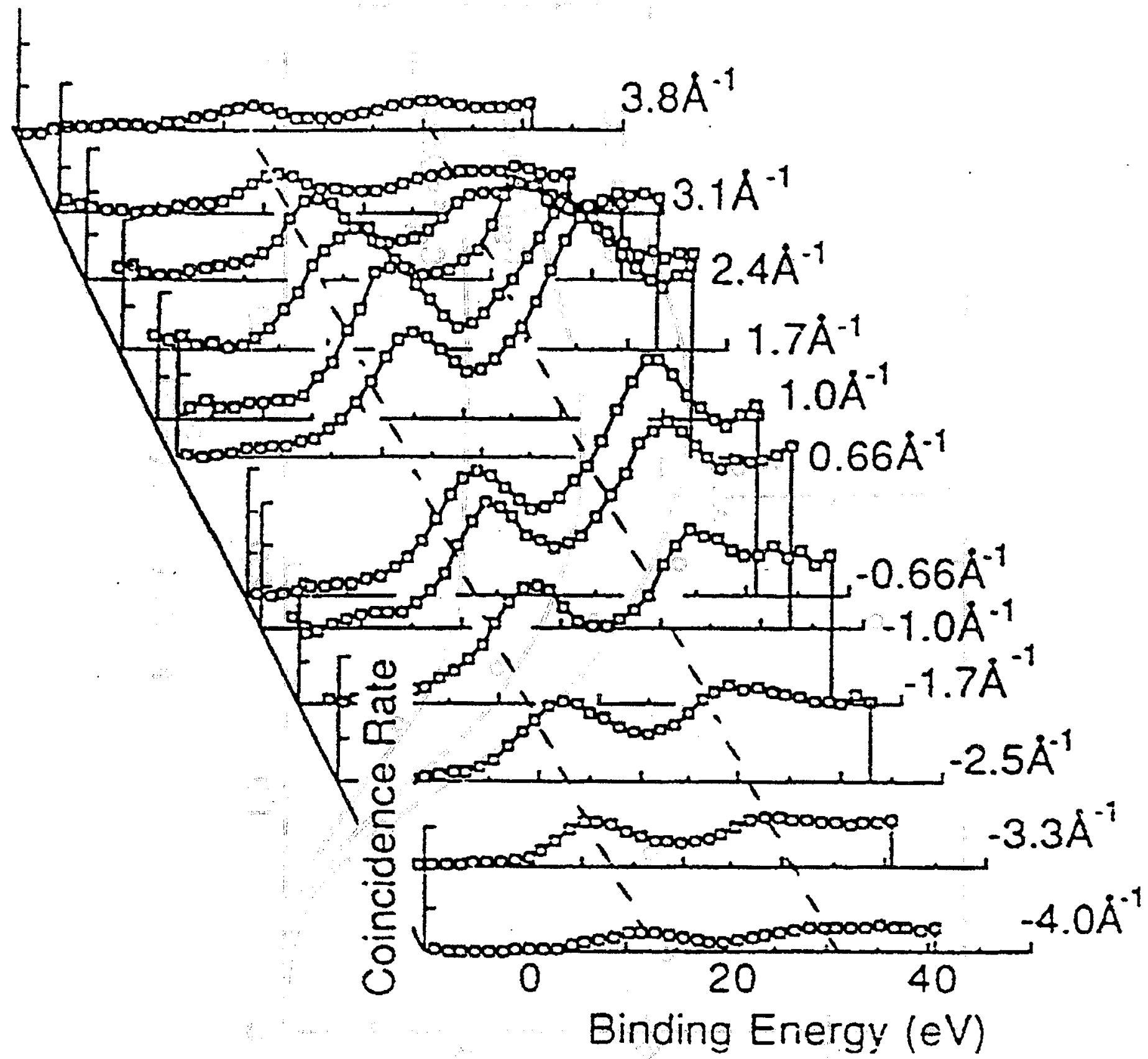

$F_{1 g}, 12$ 